\title{
LONG WAVE EXPANSIONS FOR WATER WAVES OVER RANDOM TOPOGRAPHY
}

\author{
ANNE DE BOUARD ${ }^{1}$, WALTER CRAIG ${ }^{2}$, OLIVER DÍAZ-ESPINOSA ${ }^{2}$, PHILIPPE \\ GUYENNE $^{3}$ AND CATHERINE SULEM ${ }^{4}$
}

\begin{abstract}
In this paper, we study the motion of the free surface of a body of fluid over a variable bottom, in a long wave asymptotic regime. We assume that the bottom of the fluid region can be described by a stationary random process $\beta(x, \omega)$ whose variations take place on short length scales and which are decorrelated on the length scale of the long waves. This is a question of homogenization theory in the scaling regime for the Boussinesq and KdV equations.

The analysis is performed from the point of view of perturbation theory for Hamiltonian PDEs with a small parameter, in the context of which we perform a careful analysis of the distributional convergence of stationary mixing random processes. We show in particular that the problem does not fully homogenize, and that the random effects are as important as dispersive and nonlinear phenomena in the scaling regime that is studied. Our principal result is the derivation of effective equations for surface water waves in the long wave small amplitude regime, and a consistency analysis of these equations, which are not necessarily Hamiltonian PDEs. In this analysis we compute the effects of random modulation of solutions, and give an explicit expression for the scattered component of the solution due to waves interacting with the random bottom. We show that the resulting influence of the random topography is expressed in terms of a canonical process, which is equivalent to a white noise through Donsker's invariance principle, with one free parameter being the variance of the random process $\beta$. This work is a reappraisal of the paper by Rosales \& Papanicolaou [24] and its extension to general stationary mixing processes.
\end{abstract}

\section{INTRODUCTION}

The problem of surface water waves over an uneven bottom is a classical problem of fluid mechanics, and it is relevant to coastal engineering and ocean wave dynamics. In this paper, we investigate how the presence of bottom topography affects the equations describing the limit of solutions in the long wave regime. We assume that the bottom is modeled by a stationary random process which is mixing, whose variations and whose correlation length manifest themselves on length scales that are short compared to the scale of the surface waves. In a previous work 9, we addressed the long wave limit of surface waves over a bottom which has periodic variations over short scales, in which we proved that the problem fully homogenizes. That is to say, the free surface motion can be described by a partial differential equation with constant effective coefficients, where the dependency over short scales is manifested by coefficients which are ensemble averages. Here in contrast, we show that random, realization-dependent effects are retained in the

2000 Mathematics Subject Classification. 76B15, 35Q53, 76M50, 60F17.

Key words and phrases. Water waves, random topography, long wave asymptotics. 
description of the solution. The latter paper and the present one are reappraisals and extensions of an earlier work by Rosales \& Papanicolaou [24] who address the problem through different methods.

Our approach uses a formulation in terms of perturbation theory for Hamiltonian partial differential equations, coupled with a detailed analysis of stationary ergodic processes which have mixing properties and which are considered as tempered distributions. As a first result we give an appropriate form of the Boussinesq equations. Secondly, following a series of changes of variables, we derive a system of coupled $\mathrm{KdV}$-like equations for the two components of the solution; these describe a wave propagating predominantly to the right, and a 'small' scattered wave propagating to the left. We then extract a limiting system of two effective equations through a consistency analysis. Specifically, we solve the effective system, which is composed of an equation similar to the $\mathrm{KdV}$ for the wave propagating to the right with a random component to its velocity, and a scattered wave propagating to the left. We give explicit formulas for the dominant contributions and the first corrections to this solution, quantifying the effects of the random modulation of position and amplitude. From these expressions, we compute a posteriori all the terms that have been neglected in the effective system, and prove that they are indeed of higher order. This evaluation relies on scale separation lemmas, which in turn follow from Donsker's invariance principle. Our analysis improves upon 24 in several ways. In particular we identify the canonical limiting distributions which contribute to the random asymptotic behavior of solutions, we quantify both random phase and random amplitude variations of solutions, and in addition, we extend the long-wave analysis over random topography to general stationary mixing processes.

The asymptotic system of equations that results from this analysis consists of a KdV equation with an additional linear term, and a transport equation for the scattered component driven by an inhomogeneous forcing term. The additional nonzero linear term, which either stabilizes or destabilizes solutions depending upon the sign of its coefficient, in turn depends on the statistics of the bottom variations. The presence of this term is the consequence of a subtle calculation, and to our knowledge, it has not been previously observed. In case these statistics are spatially reversible, the relevant coefficient vanishes and the equation reduces to the usual $\mathrm{KdV}$.

There has been a lot of interest in wave motion in basins with non constant bathymetry, due to its hydrodynamic importance. Recent references to the theory of linear waves include the papers of Nachbin (1995) [19], Sølna \& Papanicolaou (2000) [26, Nachbin \& Sølna (2003) [21] which discuss the theory of linear transport in a random medium. The earlier work of Howe (1971) [15] and the paper of Rosales \& Papanicolaou (1983) 24] give an asymptotic analysis of nonlinear equations of water waves. Nonlinear problems over variable topography are addressed in Nachbin (2003) [20 and Artiles \& Nachbin (2004) 2]. More recent contributions which take into account the combined effect of randomness and nonlinearity include the series of papers by Mei \& Hancock (2003) [17] and Grataloup and Mei (2003) 14] on the modulational scaling regime, and its extensions to the three dimensional case in Pihl, Mei \& Hancock (2002) [23. This work focuses on the temporal behavior of ensemble averages of solutions, giving the result that they satisfy a nonlinear Schrödinger equation with an additional dissipative term. The analog of this picture in the long wave scaling regime appears in Mei \& Li (2004) [18, where 
the bottom is assumed random but varies on the same spatial scale as the surface waves.

There is a history of rigorous analysis of the initial value problem and limiting equations in the long wave asymptotic regime of the water wave problem. Most of this work concerns the case of fluid domains with a flat bottom. The papers that address the KdV limit include Kano \& Nishida (1986) [16], Craig (1985) [7, Schneider \& Wayne (2000) [25], Wright (2005) [28] and Bona, Colin \& Lannes (2005) [5]. A recent paper which addresses specifically the Boussinesq scaling limit of the problem on a rigorous basis, and categorizes the well-posed possible limits is Bona, Chen \& Saut (2002) [4]. There has been several papers giving a rigorous analysis of the initial value problem of water waves over a variable bottom, including Yosihara (1983) [29] on the two-dimensional problem and Alvarez-Samaniego \& Lannes (2006) [1] on the two and three-dimensional problems, and a recent paper by Chazel (2007) [6]. The paper [1] considers the issue of convergence in various scaling regimes governed by long wave models. These results are in the context of a deterministic problem, with a small amplitude bottom perturbation, varying spatially on the same scale as the waves in the surface. As far as we know, there are no current rigorous analytic results for the $\mathrm{KdV}$ or Boussinesq scaling regimes in which the bottom variations occur on a short length scale, and are averaged under the nonlinear evolution of water waves.

The paper is organized as follows. Section 2 describes the problem of water waves in its Hamiltonian form, the Dirichlet-Neumann operator in the presence of a variable bottom, and the spatial scaling regime appropriate for the long wave problem. Section 3 presents the setting of stationary ergodic and mixing processes in which we work, and gives the relevant scale separation lemmas. This is the key of the paper. It furthermore gives an analysis of the natural regularization of characteristic coordinates that are applied to the KdV scaling limit. The Boussinesq regime is presented in Section 4, while the more detailed KdV regime is taken upon in Section 5. The main issue of this analysis is that the scattering of waves by the bottom variations is strong and it must be shown that the standard KdV Ansatz of unidirectional propagation remains valid despite this. The consistency analysis of the resulting asymptotic system of equation is the most detailed part of this paper. Finally, Section 6 presents some remarks on the process of ensemble averaging.

\section{HAMiLTONiAn FORMULATiON}

2.1. Hamilton equations. The time-dependent fluid domain consists of the region $S(\beta, \eta)=\left\{(x, y) \in \mathbb{R}^{n-1} \times \mathbb{R}:-h+\beta(x)<y<\eta(x, t)\right\}$, in which the fluid velocity is represented by the gradient of a velocity potential,

$$
u=\nabla \varphi, \quad \Delta \varphi=0 .
$$

The dependent variable $\eta(x, t)$ denotes the surface elevation, and $\beta(x)$ denotes the variation of the bottom of the fluid domain from its mean value. The bottom variations are chosen from a statistical ensemble $(\Omega, \mathcal{M}, \mathrm{P})$, which is indicated by the notation $\beta=\beta(x, \omega)$. The details of the ensemble and the associated probabilistic properties are described in Section 3.1

On the bottom boundary $\{y=-h+\beta(x)\}$, the velocity potential obeys Neumann boundary conditions

$$
\nabla \varphi \cdot N(\beta)=0
$$


where $N(\beta)=\left(1+\left|\partial_{x} \beta\right|^{2}\right)^{-1 / 2}\left(\partial_{x} \beta,-1\right)$ is the exterior unit normal.

The top boundary conditions are the usual kinematic and Bernoulli conditions imposed on $\{(x, y): y=\eta(x, t)\}$, namely

$$
\partial_{t} \eta=\partial_{y} \varphi-\partial_{x} \eta \cdot \partial_{x} \varphi, \quad \partial_{t} \varphi=-g \eta-\frac{1}{2}|\nabla \varphi|^{2} .
$$

The asymptotic analysis in this paper is initiated from the point of view of the perturbation theory of a Hamiltonian system with respect to a small parameter. For this purpose we describe the water wave problem as a Hamiltonian system with infinitely many degrees of freedom. In [30, Zakharov poses the equations of evolution (2.1) (2.2) (2.3) in the form of a Hamiltonian system in the canonical variables $(\eta(x), \xi(x))$ where one defines $\xi(x)=\varphi(x, \eta(x))$, the boundary values of the velocity potential on the free surface. The evolution equations take the classical form

$$
\partial_{t}\left(\begin{array}{l}
\eta \\
\xi
\end{array}\right)=\left(\begin{array}{cc}
0 & I \\
-I & 0
\end{array}\right)\left(\begin{array}{l}
\delta_{\eta} H \\
\delta_{\xi} H
\end{array}\right)=J \delta H
$$

with the Hamiltonian functional given by the expression of the total energy

$$
\begin{aligned}
H & =\iint_{-h+\beta(x)}^{\eta(x)} \frac{1}{2}|\nabla \varphi(x, y)|^{2} d y d x+\int \frac{g}{2} \eta^{2}(x) d x \\
& =\int \frac{1}{2} \xi(x) G(\beta, \eta) \xi(x) d x+\int \frac{g}{2} \eta^{2}(x) d x .
\end{aligned}
$$

The Dirichlet-Neumann operator $G(\beta, \eta)$ is the singular integral operator with which one expresses the normal derivative of the velocity potential on the free surface. It is a function of the boundary values $\xi(x)$ and of the domain itself, as parameterized by $\beta(x)$ and $\eta(x)$, which define respectively the lower and the upper boundaries of the fluid domain $S(\beta, \eta)$. That is, let $\varphi(x, y)$ satisfy the boundary value problem

$$
\begin{aligned}
\Delta \varphi & =0 \text { in } S(\beta, \eta), \\
\nabla \varphi \cdot N(\beta) & =0 \text { on the bottom boundary }\{y=-h+\beta(x)\} \\
\varphi(x, \eta(x)) & =\xi(x) \text { on the free surface }\{y=\eta(x)\} .
\end{aligned}
$$

The Dirichlet-Neumann operator is expressed as follows

$$
G(\beta, \eta) \xi(x)=\nabla \varphi(x, \eta(x)) \cdot N(\eta)\left(1+\left|\partial_{x} \eta\right|^{2}\right)^{1 / 2},
$$

where $N(\eta)$ is the exterior unit normal on the free surface. It is clearly a linear operator in $\xi$ and it is self-adjoint with this normalization. However it is nonlinear with explicitly nonlocal behavior in $\beta(x)$ and $\eta(x)$. The form of this operator, and its description in terms of $\beta$ and $\eta$ are given in the next section.

2.2. Description of $G(\beta, \eta)$. We now restrict consideration to the dimension $n=$ 2. In the undisturbed case in which the bottom is flat, the solution is formally given by a Fourier multiplier operator in the $x$-variable. Using the notation that $\partial_{x}=i D$

$$
\varphi(x, y)=\iint e^{i k\left(x-x^{\prime}\right)} \frac{\cosh (k(y+h))}{\cosh (k h)} \xi\left(x^{\prime}\right) d x^{\prime} d k=\frac{\cosh ((y+h) D)}{\cosh (h D)} \xi(x)
$$


When the bottom topography is nontrivial, as represented by $\{y=-h+\beta(x)\}$, the expression (2.8) is modified by adding a second term in order that the solution satisfies the bottom boundary conditions

$$
\varphi(x, y)=\frac{\cosh ((y+h) D)}{\cosh (h D)} \xi(x)+\sinh (y D)(L(\beta) \xi)(x) .
$$

The first term in (2.9) satisfies the homogeneous Neumann condition at $y=-h$ while the second term satisfies the homogeneous Dirichlet condition at $y=0$. The operator $L(\beta)$ in the second term acts on the boundary data $\xi(x)$ given on the free surface. In [9] we analyzed $L(\beta)$ in a nonperturbative case, where $|\beta|_{C^{1}} \sim \mathcal{O}(1)$. Here we are restricted to a perturbative regime, where we describe the expansion of the operator $G(\beta, \eta)$ for small but arbitrary perturbations $\eta(x)$ of the surface, and small bottom variations $\beta(x)$.

At order $\mathcal{O}(1)$ and $\mathcal{O}(\eta)$, one gets $G^{(0)}=D \tanh (h D)+D L(\beta)$ and $G^{(1)}=D \eta D-$ $G^{(0)} \eta G^{(0)}$. At higher order, one finds the same recursion formula for $G^{(l)}$ as for the case of a flat bottom [10] except that the role of the operator $G_{0}=D \tanh (h D)$ is now replaced by $G^{(0)}$.

Since we allow bottom perturbations to be of order $\mathcal{O}(\varepsilon)$, we will use a recursion formula given in [9] for $L(\beta)$ in powers of $\beta$.

$$
L(\beta)=L_{1}(\beta)+L_{2}(\beta)+\ldots
$$

with the first terms being

$$
\begin{aligned}
L_{1}(\beta) & =-\operatorname{sech}(h D) \beta \operatorname{sech}(h D) D \\
L_{2}(\beta) & =\operatorname{sech}(h D) \beta D \sinh (h D) L_{1} \\
& =-\operatorname{sech}(h D) \beta D \tanh (h D) \beta D \operatorname{sech}(h D) .
\end{aligned}
$$

General formulas are presented in 9 together with a Taylor expansion of the Dirichlet-Neumann operator $G(\beta, \eta)$ in powers of both $\beta$ and $\eta$. In the analysis of the present paper, we will need only the terms up to second order in $\beta$.

The Hamiltonian is thus expanded in powers of $\eta$ and $\beta$ in the form

$$
\begin{array}{r}
H(\eta, \xi ; \beta)=\frac{1}{2} \int\left(\xi D \tanh (h D) \xi+g \eta^{2}\right) d x \\
-\frac{1}{2} \int \xi D \operatorname{sech}(h D) \beta D \operatorname{sech}(h D) \xi d x \\
+\frac{1}{2} \int \xi(D \eta D-D \tanh (h D) \eta D \tanh (h D)) \xi d x \\
-\frac{1}{2} \int \xi(D \operatorname{sech}(h D) \beta D \tanh (h D) \beta D \operatorname{sech}(h D)) \xi d x \\
+\mathcal{O}\left(\beta^{3} \xi^{2}\right)+\mathcal{O}\left(\eta \beta \xi^{2}\right)+\mathcal{O}\left(\eta^{2} \xi^{2}\right) .
\end{array}
$$

By integration by parts,

$$
\begin{aligned}
& H(\eta, \xi ; \beta)=\frac{1}{2} \int\left(\xi D \tanh (h D) \xi+g \eta^{2}\right) d x-\frac{1}{2} \int \beta|D \operatorname{sech}(h D) \xi|^{2} d x \\
& \quad+\frac{1}{2} \int \xi(D \eta D-D \tanh (h D) \eta D \tanh (h D)) \xi d x \\
& \quad-\frac{1}{2} \int \overline{(D \operatorname{sech}(h D) \xi)} \beta D \tanh (h D) \beta D \operatorname{sech}(h D) \xi d x \\
& \quad+\mathcal{O}\left(\beta^{3} \xi^{2}\right)+\mathcal{O}\left(\eta \beta \xi^{2}\right)+\mathcal{O}\left(\eta^{2} \xi^{2}\right),
\end{aligned}
$$

which is the starting point for our asymptotic expansion. 
2.3. Spatial scaling and the scaled Hamiltonian. We consider the case in which the bottom varies on a short length scale, that is $\beta=\beta(x, \omega)$ is a random process, of zero mean value that satisfies ergodicity and mixing properties which will be detailed below.

The fundamental long wave scaling for the problem of surface water waves retains a balance between linear dispersive and nonlinear effects in the dynamics of the surface evolution. The scaling that anticipates this balance is through the transformation

$$
X=\varepsilon x, \quad \xi(x)=\varepsilon \tilde{\xi}(X), \quad \eta(x)=\varepsilon^{2} \tilde{\eta}(X) .
$$

As for the bottom, we assume its variations are of order $\mathcal{O}(\varepsilon)$, which are much larger that the variations of the surface elevation, namely

$$
\beta(x, \omega)=\varepsilon \tilde{\beta}(x, \omega) .
$$

We assume that $\tilde{\beta}$ is bounded in $C^{1}$ for almost every realization $\omega \in \Omega$.

In order to get the scaled Hamiltonian, we need to examine the asymptotic expansion of the Dirichlet-Neumann operator $G(\beta, \eta)$ in a multiple scale regime. We recall how formally a pseudo-differential operator acts on a multiple scale function $f(x, X)$ where $X=\varepsilon x$ (see [11] for details). In particular let $m(D)$ be a Fourier multiplier operator acting on a function $f$, defined as

$$
(m(D) f)(x)=\frac{1}{2 \pi} \int e^{i k(x-y)} m(k) f(y) d y d k .
$$

When $m(D)$ acts on a multiple scale function $f(x, X)$ with $X=\varepsilon x, D$ is replaced by $D_{x}+\varepsilon D_{X}$ and

$$
\begin{aligned}
m(D) f(x, X) & =\frac{1}{2 \pi} \int e^{i k(x-y)}\left(\sum_{j=0}^{\infty} \frac{m^{(j)}(k)}{j !} \varepsilon^{j} D_{X}^{j}\right) f(y, X) d y d k \\
& =m\left(D_{x}\right) f+\varepsilon m^{\prime}\left(D_{x}\right) D_{X} f+\cdots
\end{aligned}
$$

Applying this to the scaled Hamiltonian, we get

$$
\begin{array}{r}
H(\tilde{\eta}, \tilde{\xi} ; \tilde{\beta}, \varepsilon)=\frac{\varepsilon^{3}}{2} \int\left(h \tilde{\xi} D_{X}^{2} \tilde{\xi}+g \tilde{\eta}^{2}\right) d X \\
-\frac{\varepsilon^{4}}{2} \int \tilde{\beta}(x)\left|D_{X} \operatorname{sech}\left(\varepsilon h D_{X}\right) \tilde{\xi}\right|^{2} d X+\frac{\varepsilon^{5}}{2} \int \tilde{\xi}\left(D_{X} \tilde{\eta} D_{X} \tilde{\xi}-\frac{h^{3}}{3} D_{X}^{4} \tilde{\xi}\right) d X \\
-\frac{\varepsilon^{5}}{2} \int \frac{\left.D_{X} \operatorname{sech}\left(\varepsilon h D_{X}\right) \tilde{\xi}\right)}{\left(D_{X}\right)} \\
{\left[\tilde{\beta}(x)\left(D_{x}+\varepsilon D_{X}\right) \tanh \left(h\left(D_{x}+\varepsilon D_{X}\right)\right) \tilde{\beta}(x) D_{X} \operatorname{sech}\left(\varepsilon h D_{X}\right) \tilde{\xi}\right] d X}
\end{array}
$$

For simplicity of notation, we now drop the tildes over $\beta, \eta, \xi$. Expanding the operator $\operatorname{sech}\left(\varepsilon h D_{X}\right)$ in the second term in (2.19) gives

$$
\int \beta(x)\left|D_{X} \operatorname{sech}\left(\varepsilon h D_{X}\right) \xi\right|^{2} d X=\int \beta\left(\frac{X}{\varepsilon}\right)\left|D_{X}\left(1-\frac{1}{2} \varepsilon^{2} h^{2} D_{X}^{2}\right) \xi\right|^{2} d X .
$$

The last term of (2.19) is a little more complicated but is calculated in the same manner. Expanding $\left(D_{x}+\varepsilon D_{X}\right) \tanh \left(h\left(D_{x}+\varepsilon D_{X}\right)\right)$ we get

$$
\left(D_{x}+\varepsilon D_{X}\right) \tanh \left(h\left(D_{x}+\varepsilon D_{X}\right)\right)=D_{x} \tanh \left(h D_{x}\right)+\mathcal{O}(\varepsilon) .
$$


Finally,

$$
\begin{aligned}
& \int \overline{D_{X} \operatorname{sech}\left(\varepsilon h D_{X}\right) \xi}\left[\beta(x)\left(D_{x}+\varepsilon D_{X}\right) \tanh \left(h\left(D_{x}+\varepsilon D_{X}\right)\right) \beta(x) D_{X} \operatorname{sech}\left(\varepsilon h D_{X}\right) \xi\right] d X \\
& \quad=\int \overline{D_{X} \operatorname{sech}\left(\varepsilon h D_{X}\right) \xi}\left[\beta(x) D_{x} \tanh \left(h D_{x}\right) \beta(x)\right] D_{X} \operatorname{sech}\left(\varepsilon h D_{X}\right) \xi d X+\mathcal{O}(\varepsilon) \\
& \quad=\int\left[\beta(x) D_{x} \tanh \left(h D_{x}\right) \beta(x)\right]\left|D_{X} \xi\right|^{2} d X+\mathcal{O}(\varepsilon) .
\end{aligned}
$$

Putting all these terms together:

$$
\begin{aligned}
& H(\eta, \xi ; \beta, \varepsilon)=\frac{\varepsilon^{3}}{2} \int\left[\left(h-\varepsilon \beta(x)-\varepsilon^{2} \beta(x) D_{x} \tanh \left(h D_{x}\right) \beta(x)\right)\left|D_{X} \xi\right|^{2}+g \eta^{2}\right. \\
& \left.\quad+\frac{\varepsilon^{2}}{2}\left(\xi D_{X} \eta D_{X} \xi-\frac{h^{3}}{3} \xi D_{X}^{4} \xi\right)\right] d X+o\left(\varepsilon^{5}\right) .
\end{aligned}
$$

\section{Homogenization AND SCALE SEPARATion}

The purpose of this section is to understand the asymptotic behavior of integrals of the form

$$
\int_{-\infty}^{+\infty} \gamma\left(\frac{X}{\varepsilon}\right) f(X) d X:=Z_{\varepsilon}(\gamma, f)
$$

where $f(X)$ comes from expressions which involve the physical variables which depend only upon large spatial scales, and where $\gamma(x)=\gamma(x ; \omega)$ is a stationary ergodic process taken from the statistical ensemble $\Omega$ from which our realizations of the bottom are sampled. Principle examples of such integral expressions in the Hamiltonian for water waves are

$$
\int_{-\infty}^{+\infty} \beta\left(\frac{X}{\varepsilon} ; \omega\right)\left|D_{X} \xi(X)\right|^{2} d X
$$

as well as

$$
\int_{-\infty}^{+\infty}\left(\beta D_{x} \tanh \left(h D_{x}\right) \beta\right)\left(\frac{X}{\varepsilon}\right)\left|D_{X} \xi(X)\right|^{2} d X .
$$

In our previous work 9, expressions of this form are analyzed under the hypothesis that $\beta$ was a periodic function of $x$. In the present paper, we are concerned with the case in which the bottom variations $\beta(x, \omega)$ are decorrelated over large spatial scales, which is quantified with a mixing condition on $\Omega$.

3.1. Stationary ergodic processes and mixing. We take our statistical ensemble of random bottom variations of the fluid domain to be modeled by a stationary ergodic process which will possess some properties of mixing. Mathematically, given a probability space $(\Omega, \mathcal{M}, \mathrm{P})$ equipped with a group of $\mathrm{P}$-measure preserving translations $\left\{\tau_{y}: y \in \mathbb{R}\right\}$, and a function $G: \Omega \rightarrow \mathbb{R}$, then a stationary process $\gamma$ is given by $\gamma(x ; \omega):=G\left(\tau_{x} \omega\right)$. The notation for the probability of a set $A \in \mathcal{M}$ is $\mathrm{P}(A)$, and integrals of functions $F$ over this probability space are denoted by

$$
\int_{\Omega} F d P=\mathrm{E}(F)
$$

We further require that the measure be ergodic with respect to $\left\{\tau_{y}\right\}_{y \in \mathbb{R}}$, meaning that for any bounded measurable function $F: \Omega \rightarrow \mathbb{R}$, then for P-almost every 
realization $\omega$,

$$
\lim _{L \rightarrow \infty} \frac{1}{L} \int_{0}^{L} F\left(\tau_{y} \omega\right) d y=\mathrm{E}(F) .
$$

For our purposes, we would like to take $\Omega:=C(\mathbb{R})$ the space of bounded continuous functions, for which the one-parameter group of translations is just that, $\left(\tau_{y} \gamma\right)(\cdot)=\gamma(\cdot+y)$, for $y \in \mathbb{R}$. However it turns out that our sample space $C(\mathbb{R})$ must be enlarged to a subset of the space of tempered distributions $\mathcal{S}^{\prime}$, as the process of taking limits invokes Donsker's invariance principle, and the support of our limiting measures is on distributions corresponding to one (or several) derivatives of Brownian motion. The modeling of a random bottom will require properties of asymptotic independence of typical realizations with respect to the probability measure $(\mathcal{M}, \mathrm{P})$, specifically that the translations $\left\{\tau_{y}\right\}_{y \in \mathbb{R}}$ exhibit a mixing property with respect to it. There are several notions of mixing in the literature [13. For simplicity, we adopt the notion of uniform strong mixing (called $\alpha$-mixing), although weaker conditions would also work in our setting. The stationary process defines a natural filtration on the probability space given by the $\sigma$-algebras $\mathcal{M}_{v}^{u}=\sigma(\gamma(y, \omega): v \leq y \leq u)$. The notion of $\alpha$-mixing is that there is a bounded function $\alpha(y)$ for which $\alpha(y) \rightarrow 0$ as $y \rightarrow \infty$ such that for any two sets $A \in \mathcal{M}_{0}^{\infty}$ and $B \in \mathcal{M}_{-\infty}^{0}$ then

$$
\left|\mathrm{P}\left(A \cap \tau_{y}(B)\right)-\mathrm{P}(A) \mathrm{P}(B)\right|<\alpha(y) .
$$

Note that mixing implies the process is ergodic. So that Donsker's invariance principle will extend to this mixing process [22, we require that $\alpha(y)=\mathcal{O}(1 / y \log (y))$ for $y \mapsto+\infty$ as well as

$$
\int_{0}^{\infty} \alpha(y) d y<+\infty
$$

The integral (3.3) involves a nonlocal expression in the bottom variations $\beta(x)$, implying that the random processes we are led to analyse will never be perfectly decorrelated under any finite translation. Indeed, the spatial decay of the kernel of the operator $D \tanh (h D)$ implies a lower bound on $\alpha(y)$ of the form

$$
\alpha(y)>e^{-2 h y},
$$

even for statistics of the actual realizations of the bottom variations $\beta(x, \omega)$ which are fully decorrelated under sufficiently large finite translations $|y|>R$.

For the zero mean process $\gamma$, define the covariance function $\rho_{\gamma}$ to be

$$
\rho_{\gamma}(y):=\mathrm{E}(\gamma(0 ; \omega) \gamma(y ; \omega))=\mathrm{E}\left(\gamma(0 ; \omega) \tau_{y} \gamma(0 ; \omega)\right),
$$

which is an even function of $y$ ([12] page 123, or [3], page 178). The variance $\sigma_{\gamma}^{2}$ is given by the expression

$$
\sigma_{\gamma}^{2}:=2 \int_{0}^{\infty} \rho_{\gamma}(y) d y
$$

The integral exists because of the hypothesis of mixing of the underlying process. The variance can take on any value in $[0,+\infty)$, and we are principally concerned with the situation in which $\sigma_{\gamma}>0$. To this end we note the following fact.

Lemma 3.1. When the process $\beta(x, \omega)=\partial_{x} \gamma(x, \omega)$, for $\gamma(x) \in C^{1}$, a zero-mean, stationary process with the above mixing properties, then

$$
\sigma_{\beta}=0 \text {. }
$$


Proof. By definition,

$$
\begin{aligned}
\sigma_{\beta}^{2} & =2 \int_{0}^{+\infty} \mathrm{E}(\beta(0) \beta(y)) d y=2 \int_{0}^{+\infty} \mathrm{E}(\beta(x) \beta(x+y)) d y \\
& =2 \int_{0}^{+\infty} \mathrm{E}\left(\partial_{x} \gamma(x) \partial_{x} \gamma(x+y)\right) d y=2 \int_{0}^{+\infty} \mathrm{E}\left(\partial_{x} \gamma(x) \partial_{y} \gamma(x+y)\right) d y \\
& =2 \int_{0}^{+\infty} \partial_{y} \mathrm{E}\left(\partial_{x} \gamma(x) \gamma(x+y)\right) d y .
\end{aligned}
$$

Therefore by integrating,

$$
\sigma_{\beta}^{2}=-2 \mathrm{E}\left(\partial_{x} \gamma(x) \gamma(x)\right)+\left.2 \mathrm{E}\left(\partial_{x} \gamma(x) \gamma(x+y)\right)\right|_{y=+\infty}=-\mathrm{E}\left(\partial_{x} \gamma^{2}(x)\right),
$$

because the process is mixing. Using the hypothesis of ergodicity,

$$
\mathrm{E}\left(\partial_{x} \gamma^{2}(x)\right)=\lim _{T \rightarrow \infty} \frac{1}{T} \int_{0}^{T} \partial_{x} \gamma^{2}(x) d x=\left.\lim _{T \rightarrow \infty} \frac{1}{T}\left(\gamma^{2}(x)\right)\right|_{x=0} ^{T}=0 .
$$

Thus the most interesting processes are those which are not derived from derivatives of another stationary process; this fact will be reflected in our analysis of the asymptotics of the integrals (3.1) in the next section.

3.2. Scale separation. The asymptotic analysis of Hamiltonians or partial differential equations which involve random coefficients needs to establish a clear criterion with which to characterize terms by their order parameter. In our present analysis, we view each term as a tempered distribution in space and time, namely in $\mathcal{S}^{\prime}\left(\mathbb{R}^{2}\right)$. We consider a term $a(X, t ; \varepsilon)$ to be of $\operatorname{order} \mathcal{O}\left(\varepsilon^{r}\right)$ if for any Schwartz class test func-

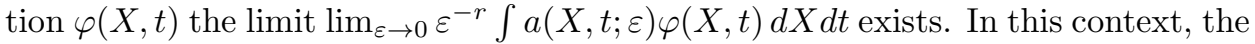
terms of a partial differential equation with random coefficients represent random ensembles of tempered distributions, say $\{a(X, t ; \omega, \varepsilon): \omega \in \Omega\} \subseteq \mathcal{S}^{\prime}\left(\mathbb{R}^{2}\right)$, which we state to be of order $\mathcal{O}\left(\varepsilon^{r}\right)$ if for any test function $\varphi(X, t) \in \mathcal{S}\left(\mathbb{R}^{2}\right)$ the probability measures $d \mathrm{P}_{\varepsilon}$ of $\varepsilon^{-r} \int a(X, t ; \varepsilon, \omega) \varphi(X, t) d X d t$ converges weakly to some $d \mathrm{P}_{0}$. In this section we discuss the behavior of such terms in the form

$$
\begin{gathered}
\int \gamma\left(\frac{X}{\varepsilon}, t ; \omega\right) v(X, t) \varphi(X, t) d X d t \\
\int \gamma_{1}\left(\frac{X}{\varepsilon}, t ; \omega\right) \gamma_{2}\left(\frac{X+c t}{\varepsilon}, t ; \omega\right) v(X, t) \varphi(X, t) d X d t
\end{gathered}
$$

where $\gamma$ is a stationary mixing process, $v$ is a solution to one of the several differential equations under discussion, and $\varphi$ plays the rôle of a test function.

Lemma 3.2. For $\gamma(x ; \omega)$ a stationary ergodic process and for $f(X) \in L^{1}(\mathbb{R})$, then for $\mathrm{P}$-a.e. realization $\omega$,

$$
\int_{-\infty}^{+\infty} f(X) \gamma\left(\frac{X}{\varepsilon} ; \omega\right) d X=\mathrm{E}(\gamma) \int_{-\infty}^{+\infty} f(X) d X+o(1) .
$$

Proof. For a Schwartz class function $f$ we have

$$
\begin{gathered}
\int_{-\infty}^{+\infty} f(X) \gamma\left(\frac{X}{\varepsilon} ; \omega\right) d X=\varepsilon \int_{-\infty}^{+\infty} f(X) \frac{d}{d X}\left(\int_{0}^{\frac{X}{\varepsilon}} \gamma(s ; \omega) d s\right) d X \\
=-\int_{-\infty}^{+\infty} X f^{\prime}(X) \frac{\varepsilon}{X} \int_{0}^{\frac{X}{\varepsilon}} \gamma(s ; \omega) d s d X .
\end{gathered}
$$


As $\varepsilon \rightarrow 0$, combining Birkhoff ergodic theorem

$$
\frac{\varepsilon}{X} \int_{0}^{\frac{X}{\varepsilon}} \gamma(s ; \omega) d s \rightarrow \mathrm{E}(\gamma)
$$

with the dominated convergence theorem leads to

$$
\int_{-\infty}^{+\infty} f(X) \gamma\left(\frac{X}{\varepsilon} ; \omega\right) d X \rightarrow-\mathrm{E}(\gamma) \int_{-\infty}^{+\infty} X f^{\prime}(X) d X
$$

and finally (3.12). In fact it suffices that $f \in L^{1}(\mathbb{R})$ for the result to hold.

The immediate application of the lemma is to the integrals (3.2) (3.3), at least to the order implied by Lemma 3.2 for their mean values. Under the assumption that $\xi(X) \in H^{1}(\mathbb{R})$, the first of these vanishes up to order $o(1)$ as $\mathrm{E}(\beta)=0$, at least for P-a.e. realization $\omega$. What is clear is that the fluctuations of (3.2) will play an important rôle in the derivation of the appropriate Hamiltonian equations of motion. The second integral (3.3) is less straightforward, as the mixing condition (3.7) is in competition with the integral operators represented by the Fourier multiplier operators of the expression. We have that

$$
\begin{aligned}
& \left.\int\left(\beta(x) D_{x} \tanh \left(h D_{x}\right) \beta(x)\right)\right|_{x=\frac{X}{\varepsilon}}\left|D_{X} \xi(X)\right|^{2} d X \\
& \rightarrow \mathrm{E}\left(\beta D_{x} \tanh \left(h D_{x}\right) \beta\right) \int\left|D_{X} \xi(X)\right|^{2} d X .
\end{aligned}
$$

There are two things to discuss with this statement. The first is that whenever $\gamma(x, \omega) \in C^{1}$ is stationary with regard to some probability space $(\Omega, \mathcal{M}, \mathrm{P})$, then an order zero Fourier multiplier operator applied to $\gamma(x)$ is also stationary. Indeed, translation is respected

$$
\begin{aligned}
m\left(D_{x}\right) \gamma\left(x, \tau_{y} \omega\right) & =\frac{1}{2 \pi} \int e^{i k\left(x-x^{\prime}\right)} m(k) \gamma\left(x^{\prime}, \tau_{y} \omega\right) d x^{\prime} d k \\
& =\frac{1}{2 \pi} \int e^{i k\left(x-x^{\prime}\right)} m(k) \gamma\left(x^{\prime}-y, \omega\right) d x^{\prime} d k \\
& =\frac{1}{2 \pi} \int e^{i k\left((x-y)-x^{\prime}\right)} m(k) \gamma\left(x^{\prime}, \omega\right) d x^{\prime} d k \\
& =m\left(D_{x}\right) \gamma(x-y, \omega) .
\end{aligned}
$$

Furthermore, continuous functions of $\gamma \in C^{1}$, such as $g(\gamma)=\left(\gamma m\left(D_{x}\right) \gamma\right)(0)$ are measurable. By the ergodic theorem, for any bounded measurable $F$

$$
\lim _{L \rightarrow \infty} \frac{1}{L} \int_{0}^{L} F\left(\tau_{x} g(\gamma)\right) d x=\mathrm{E}(F(g))
$$

and therefore the process $\tau_{x} g(\gamma)$ is ergodic. Secondly, the expectation values of quadratic functions of $\gamma$ may be computed from the covariance function $\rho_{\gamma}$ of the stationary process. For example,

$$
\begin{aligned}
& \mathrm{E}\left(\gamma m\left(D_{x}\right) \gamma\right)=\lim _{y \rightarrow 0} \mathrm{E}\left(\gamma(x) m\left(D_{x}\right) \gamma(x-y)\right) \\
& =\lim _{y \rightarrow 0} \mathrm{E}\left(m\left(-D_{y}\right) \gamma(x) \gamma(x-y)\right)=\lim _{y \rightarrow 0} m\left(-D_{y}\right) \rho_{\gamma}(y) \\
& =m\left(-D_{y}\right) \rho_{\gamma}(0) .
\end{aligned}
$$

Using these two facts, (3.16) is verified as the principal contribution from integral (3.3). 
Lemma 3.3. 3] Suppose that $\beta(x ; \omega)$ is a stationary ergodic process which is mixing, with a rate $\alpha(y)$ which satisfies the condition (3.7). Assume that $\mathrm{E}(\beta)=0$ and that $\sigma_{\beta} \neq 0$. Define

$$
Y_{\varepsilon}(\beta)(X)=\frac{\sqrt{\varepsilon}}{\sigma_{\beta}} \int_{0}^{\frac{X}{\varepsilon}} \beta(y) d y .
$$

As $\varepsilon$ tends to zero, we have, in the sense convergence in law that

$$
Y_{\varepsilon}(\beta)(X) \rightarrow B(X),
$$

where $B_{\omega}(X)=B(X)$ is a normalized Brownian motion.

In particular, let $f(X) \in \mathcal{S}$ be a Schwartz class function, then

$$
\begin{aligned}
& \frac{1}{\sigma_{\beta} \sqrt{\varepsilon}} Z_{\varepsilon}(\beta, f):=\int_{-\infty}^{+\infty} \frac{1}{\sigma_{\beta} \sqrt{\varepsilon}} \beta\left(\frac{X}{\varepsilon}\right) f(X) d X \\
& =\int_{-\infty}^{+\infty} Y_{\varepsilon}^{\prime}(\beta)(X) f(X) d X=\int_{-\infty}^{+\infty}-\partial_{X} f(X) B(X) d X+o(1) .
\end{aligned}
$$

This is to say that under the mild condition of mixing given in (3.7), the integrals in question converge to a canonical stationary process, for which only two parameters are distinguished, the mean value $\mathrm{E}(\beta)$ and the variance $\sigma_{\beta}^{2}$. This canonical process is given by white noise,

$$
\int_{-\infty}^{+\infty} \beta\left(\frac{X}{\varepsilon}\right) f(X) d X=\int_{-\infty}^{+\infty}\left(\mathrm{E}(\beta)+\sqrt{\varepsilon} \sigma_{\beta} \partial_{X} B(X)\right) f(X) d X+o(\sqrt{\varepsilon}),
$$

where the equality is in the sense of convergence in law. The function $f(X)$ in the integrand must be sufficiently smooth for the latter quantities to have a mathematical sense. In fact we consider the operation of multiplication by $\beta\left(\frac{X}{\varepsilon}\right)$ to be in the distributional sense, which has for a limit the distribution $\sqrt{\varepsilon} \sigma_{\beta} \partial_{X} B(X) \in \mathcal{S}^{\prime}$. This is given a precise statement in the following lemma.

Lemma 3.4. As a distribution, multiplication by $\beta(X / \varepsilon)$ has a canonical limit in $\mathcal{S}^{\prime}$. Indeed, for $f \in \mathcal{S}$,

$$
\beta\left(\frac{X}{\varepsilon}\right) f(X)=\mathrm{E}(\beta) f(X)+\sqrt{\varepsilon} \sigma_{\beta} \partial_{X} B(X) f(X)+o(\sqrt{\varepsilon}) .
$$

Proof. Test the quantity above with a Schwartz class function $\varphi(X)$;

$$
\begin{aligned}
\int & \beta\left(\frac{X}{\varepsilon}\right) f(X) \varphi(X) d X \\
= & \mathrm{E}(\beta) \int(f(X) \varphi(X)) d X-\sqrt{\varepsilon} \sigma_{\beta} \int B(X) \partial_{X}(f \varphi) d X+o(\sqrt{\varepsilon}) \\
= & \int\left(\mathrm{E}(\beta)+\sqrt{\varepsilon} \sigma_{\beta} \partial_{X} B(X)\right) f(X) \varphi(X) d X+o(\sqrt{\varepsilon}) .
\end{aligned}
$$

This is to say that for each $f$, the random variable $Z_{\varepsilon}(\beta, f)$ given in (3.22) is asymptotically normally distributed. Given two functions $f, g \in \mathcal{S}$, the covariance function $\mathrm{E}\left(Z_{\varepsilon}(\beta, f) Z_{\varepsilon}(\beta, g)\right)$ can be computed in the limit as $\varepsilon \rightarrow 0$. Indeed

$$
\begin{aligned}
\mathrm{E} & \left(Z_{\varepsilon}(\beta, f) Z_{\varepsilon}(\beta, g)\right)=\frac{1}{\varepsilon} \iint \rho_{\beta}\left(\frac{X-X^{\prime}}{\varepsilon}\right) f(X) g\left(X^{\prime}\right) d X d X^{\prime} \\
& =\iint \rho_{\beta}\left(x^{\prime}\right) f(X) g\left(X-\varepsilon x^{\prime}\right) d X d x^{\prime} \\
& =\iint \rho_{\beta}\left(x^{\prime}\right) f(X)\left(g(X)-\varepsilon x^{\prime} \partial_{X} g(X)+\frac{\varepsilon^{2}}{2} x^{\prime 2} \partial_{X}^{2} g(X)+\ldots\right) d X d x^{\prime} .
\end{aligned}
$$


Noting that the term at order $\varepsilon$ vanishes because $\rho_{\beta}$ is an even function, we have

$$
\begin{gathered}
\mathrm{E}\left(Z_{\varepsilon}(\beta, f) Z_{\varepsilon}(\beta, g)\right)=\int \rho_{\beta}\left(x^{\prime}\right) d x^{\prime} \int f(X) g(X) d X \\
-\frac{\varepsilon^{2}}{2} \int x^{\prime 2} \rho_{\beta}\left(x^{\prime}\right) d x^{\prime} \int \partial_{X} f(X) \partial_{X} g(X) d X+\ldots
\end{gathered}
$$

In the limit as $\varepsilon \rightarrow 0$, this quantity converges to

$$
\mathrm{E}\left(Z_{0}(f) Z_{0}(g)\right)=\sigma_{\beta}^{2} \int f(X) g(X) d X,
$$

where $Z_{0}(f)=\sigma_{\beta} \sqrt{\varepsilon} \int f(X) \partial_{X} B(X) d X$. This expression is consistent with the covariance of the white noise process being given by $\sigma_{\beta}^{2} \delta\left(X-X^{\prime}\right)$.

In the case of a process $\beta(x)$ for which $\sigma_{\beta}=0$, the limit process for $Y_{\varepsilon}(X)$ is of a different character. In particular, consider a stationary mixing process which is the derivative of another stationary process. Indeed let $\gamma(x) \in C^{r+1}(\mathbb{R})$, and set $\beta(x)=\partial_{x}^{r} \gamma(x)$. Automatically $\mathrm{E}(\beta)=0$ and $\sigma_{\beta}=0$. In this situation we have a different asymptotic result for the behavior of integrals such as in (3.1).

Lemma 3.5. Suppose that $\gamma(x) \in C^{r+1}(\mathbb{R})$ is a stationary ergodic process which satisfies the mixing condition (3.7), and set $\beta(x)=\partial_{x}^{r} \gamma(x)$. Then the process $\beta(X / \varepsilon)$ is asymptotic in the sense of distributions to higher derivatives of Brownian motion. That is, for $\varphi(X) \in \mathcal{S}$ we have

$$
\int \beta\left(\frac{X}{\varepsilon}\right) \varphi(X) d X=\varepsilon^{r+1 / 2} \sigma_{\gamma} \int \partial_{X}^{r+1} B(X) \varphi(X) d X+o\left(\varepsilon^{r+1 / 2}\right) .
$$

Proof. Using $\varphi(X)$ as a test function,

$$
\begin{aligned}
\int \beta\left(\frac{X}{\varepsilon}\right) \varphi(X) d X & =\int \partial_{x}^{r} \gamma\left(\frac{X}{\varepsilon}\right) \varphi(X) d x \\
& =(-1)^{r} \varepsilon^{r} \int \gamma\left(\frac{X}{\varepsilon}\right) \partial_{X}^{r} \varphi(X) d X \\
& =(-1)^{r+1} \varepsilon^{r+1 / 2} \sigma_{\gamma} \int Y_{\varepsilon}(\gamma)(X) \partial_{X}^{r+1} \varphi(X) d X \\
& =\varepsilon^{r+1 / 2} \sigma_{\gamma} \int \partial_{X}^{r+1} B(X) \varphi(X) d X+o\left(\varepsilon^{r+1 / 2}\right) .
\end{aligned}
$$

There are further technical results that we will use repeatedly in the analysis of the equations in the $\mathrm{KdV}$ asymptotic regime, having to do with limits in the sense of tempered distributions of products of scaled processes. In this context, consider $\gamma=\left(\gamma_{1}, \gamma_{2}\right)$ a vector of stationary processes which satisfy the mixing conditions (3.6) (3.7). Consider their product $\gamma_{1}(X / \varepsilon) \gamma_{2}((X+c t) / \varepsilon)$ for some nonzero constant $c$ as a tempered distribution in the limit $\varepsilon \rightarrow 0$. Define the covariance matrix of the vector process by

where

$$
C(\gamma)=\left(\begin{array}{cc}
\sigma_{1}^{2} & \rho_{12} \\
\rho_{12} & \sigma_{2}^{2}
\end{array}\right)
$$

$$
\sigma_{j}^{2}=2 \int_{0}^{\infty} \mathrm{E}\left(\gamma_{j}(0) \gamma_{j}(y)\right) d y, \quad \rho_{12}=\rho_{21}=\int_{-\infty}^{\infty} \mathrm{E}\left(\gamma_{1}(0) \gamma_{2}(y)\right) d y .
$$


Lemma 3.6. If the vector process $\gamma=\left(\gamma_{1}, \gamma_{2}\right)$ is stationary and satisfies the mixing conditions (3.6) (3.7), then the process

$$
Y_{\varepsilon}(\gamma)=\sqrt{\varepsilon}\left(\int_{0}^{\frac{X}{\varepsilon}} \gamma_{1}(y) d y, \int_{0}^{\frac{X}{\varepsilon}} \gamma_{2}(y) d y\right)
$$

converges to the two-dimensional Brownian motion $B(X)=\left(B_{1}(X), B_{2}(X)\right)$ with covariance matrix $C(\gamma)$.

This result is analogous to Lemma 3.3 in the vector process case. From it, we derive the next useful result on products of two mixing processes.

Lemma 3.7. Suppose that $\left(\beta_{1}(x), \beta_{2}(x)\right)$ is a $C^{1}(\mathbb{R})$ vector stationary ergodic process which satisfies the mixing condition (3.6) (3.7), and let $c$ be a nonzero constant. The new process formed by the product $\varepsilon^{-1} \beta_{1}(X / \varepsilon) \beta_{2}((X+c t) / \varepsilon)$ converges in the sense of distributions on space-time to products of derivatives of a pair of Brownian motions with covariance matrix $C(\beta)$. More precisely, for a test function $\varphi(X, t) \in \mathcal{S}$ then

$$
\begin{gathered}
\int \beta_{1}\left(\frac{X}{\varepsilon}\right) \beta_{2}\left(\frac{X+c t}{\varepsilon}\right) \varphi(X, t) d X d t \\
=\varepsilon \int \partial_{X} B_{1}(X) \partial_{X} B_{2}(X+c t) \varphi(X, t) d X d t+o(\varepsilon),
\end{gathered}
$$

where the covariance matrix of $\left(B_{1}(X), B_{2}(X)\right)$ is given by $C(\beta)$. In case $\beta_{j}=$ $\partial_{x}^{r_{j}} \gamma_{j}$ for indices $j=1,2$, with $\gamma_{j} \in C^{r_{j}+1}(\mathbb{R})$ (so that $\sigma_{\beta_{j}}=0$ if $r_{j} \neq 0$ ) the new process satisfies

$$
\begin{gathered}
\int \beta_{1}\left(\frac{X}{\varepsilon}\right) \beta_{2}\left(\frac{X+c t}{\varepsilon}\right) \varphi(X, t) d X d t \\
=\varepsilon^{r_{1}+r_{2}+1} \int \partial_{X}^{r_{1}+1} B_{1}(X) \partial_{X}^{r_{2}+1} B_{2}(X+c t) \varphi(X, t) d X d t+o\left(\varepsilon^{r_{1}+r_{2}+1}\right),
\end{gathered}
$$

where $\left(B_{1}(X), B_{2}(X)\right)$ are $C(\gamma)$-correlated.

Proof. Start with the case in which both $\sigma_{\beta_{j}}$ are nonzero, and write

$$
\begin{aligned}
& \int \beta_{1}\left(\frac{X}{\varepsilon}\right) \beta_{2}\left(\frac{X+c t}{\varepsilon}\right) \varphi(X, t) d X d t=\int \beta_{1}\left(\frac{X}{\varepsilon}\right) \beta_{2}\left(\frac{X^{\prime}}{\varepsilon}\right) \varphi\left(X, \frac{X^{\prime}-X}{c}\right) \frac{d X d X^{\prime}}{c} \\
& =\varepsilon^{2} \int \partial_{X}\left(\int_{0}^{\frac{X}{\varepsilon}} \beta_{1}(\tau) d \tau\right) \partial_{X^{\prime}}\left(\int_{0}^{\frac{X^{\prime}}{\varepsilon}} \beta_{2}\left(\tau^{\prime}\right) d \tau^{\prime}\right) \varphi\left(X, \frac{X^{\prime}-X}{c}\right) \frac{d X d X^{\prime}}{c} \\
& =\varepsilon \int\left(\sqrt{\varepsilon} \int_{0}^{\frac{X}{\varepsilon}} \beta_{1}(\tau) d \tau\right)\left(\sqrt{\varepsilon} \int_{0}^{\frac{X^{\prime}}{\varepsilon}} \beta_{2}\left(\tau^{\prime}\right) d \tau^{\prime}\right) \partial_{X} \partial_{X^{\prime}} \varphi\left(X, \frac{X^{\prime}-X}{c}\right) \frac{d X d X^{\prime}}{c} .
\end{aligned}
$$

The latter expression is a continuous function of the processes $Y_{\varepsilon}(\beta)=\left(Y_{\varepsilon}\left(\beta_{1}\right), Y_{\varepsilon}\left(\beta_{2}\right)\right)$ of equation (3.20), which itself converges in law to two-dimensional Brownian motion with covariance matrix $C(\beta)$ as described by Donsker's invariance principle. Therefore the asymptotic expression for (3.31) is given by

$$
\varepsilon \int \partial_{X} B_{1}(X) \partial_{X} B_{2}(X+c t) \varphi(X, t) d X d t
$$

where $B_{1}(X)$ and $B_{2}(X)$ are two copies of Brownian motions with the correlation matrix $C(\beta)$. The general case reduces to the above particular case through integrations by parts. Indeed

$$
\begin{array}{r}
\int \beta_{1}\left(\frac{X}{\varepsilon}\right) \beta_{2}\left(\frac{X+c t}{\varepsilon}\right) \varphi(X, t) d X d t \\
=\varepsilon^{r_{1}+r_{2}} \int \partial_{X}^{r_{1}} \gamma_{1}\left(\frac{X}{\varepsilon}\right) \partial_{X}^{r_{2}} \gamma_{2}\left(\frac{X+c t}{\varepsilon}\right) \varphi(X, t) d X d t
\end{array}
$$




$$
=(-1)^{r_{1}+r_{2}} \frac{\varepsilon^{r_{1}+r_{2}}}{c^{r_{2}}} \int \gamma_{1}\left(\frac{X}{\varepsilon}\right) \gamma_{2}\left(\frac{X+c t}{\varepsilon}\right) \partial_{X}^{r_{1}} \partial_{t}^{r_{2}} \varphi(X, t) d X d t,
$$

which reduces the problem to the previous case.

There is another integral that needs to be evaluated in our further analysis. It has the form

$$
\int_{X, t} \int_{X}^{X+\sqrt{g h t}} \beta\left(\frac{X}{\varepsilon}\right) \beta\left(\frac{\theta}{\varepsilon}\right) \varphi(\theta, X, t) d \theta d X d t .
$$

The next lemma shows that such integrals have probability measures whose weak limits converge with order at least $\mathcal{O}(\varepsilon)$.

Lemma 3.8. Suppose that $\left(\beta_{1}(x), \beta_{2}(x)\right)$ is a $C^{1}(\mathbb{R})$ vector stationary ergodic process which satisfy the mixing conditions (3.6) and (3.7). For test functions $\varphi(\theta, X, t) \in \mathcal{S}$,

$$
\int d X d t \int_{X}^{X+\sqrt{g h t}}\left[\beta_{1}\left(\frac{X}{\varepsilon}\right) \beta_{2}\left(\frac{\theta}{\varepsilon}\right)+\beta_{2}\left(\frac{X}{\varepsilon}\right) \beta_{1}\left(\frac{\theta}{\varepsilon}\right)\right] \varphi(\theta, X, t) d \theta=\mathcal{O}(\varepsilon) .
$$

Proof. The integral is written as the sum of two terms, each one of the form

$$
\begin{gathered}
\int d X d t \int_{X}^{X+\sqrt{g h t} t} \beta_{i}\left(\frac{X}{\varepsilon}\right) \beta_{j}\left(\frac{\theta}{\varepsilon}\right) \varphi(\theta, X, t) d \theta \\
=\varepsilon \int d X d t \int_{X}^{X+\sqrt{g h} t} \partial_{X}\left(\sqrt{\varepsilon} \int_{0}^{\frac{X}{\varepsilon}} \beta_{i}(s) d s\right) \partial_{\theta}\left(\sqrt{\varepsilon} \int_{0}^{\frac{\theta}{\varepsilon}} \beta_{j}(s) d s\right) \varphi(\theta, X, t) d \theta \\
=\varepsilon \int d X d t \int_{X}^{X+\sqrt{g h} t}\left(\sqrt{\varepsilon} \int_{0}^{\frac{X}{\varepsilon}} \beta_{i}(s) d s\right)\left(\sqrt{\varepsilon} \int_{0}^{\frac{\theta}{\varepsilon}} \beta_{j}(s) d s\right) \partial_{X \theta} \varphi(\theta, X, t) d \theta \\
+\varepsilon \int d X d t\left(\sqrt{\varepsilon} \int_{0}^{\frac{X}{\varepsilon}} \beta_{i}(s) d s\right)\left[\left(\sqrt{\varepsilon} \int_{0}^{\frac{X+\sqrt{g h} t}{\varepsilon}} \beta_{j}(s) d s\right) \partial_{\theta} \varphi(X+\sqrt{g h} t, X, t)\right. \\
\left.-\left(\sqrt{\varepsilon} \int_{0}^{\frac{X}{\varepsilon}} \beta_{j}(s) d s\right) \partial_{\theta} \varphi(X, X, t)\right] \\
-\varepsilon \int d X d t\left(\sqrt{\varepsilon} \int_{0}^{\frac{X}{\varepsilon}} \beta_{i}(s) d s\right)\left[\left(\sqrt{\varepsilon} \int_{0}^{\frac{X+\sqrt{g h t}}{\varepsilon}} \beta_{j}(s) d s\right) \partial_{X} \varphi(X+\sqrt{g h} t, X, t)\right. \\
\left.-\left(\sqrt{\varepsilon} \int_{0}^{\frac{X}{\varepsilon}} \beta_{j}(s) d s\right) \partial_{X} \varphi(X, X, t)\right] \\
-\varepsilon \int d X d t\left(\sqrt{\varepsilon} \int_{0}^{\frac{X}{\varepsilon}} \beta_{i}(s) d s\right)\left[\frac{1}{\sqrt{\varepsilon}} \beta_{j}\left(\frac{X+\sqrt{g h} t}{\varepsilon}\right) \varphi(X+\sqrt{g h} t, X, t)\right. \\
\left.-\frac{1}{\sqrt{\varepsilon}} \beta_{j}\left(\frac{X}{\varepsilon}\right) \varphi(X, X, t)\right]
\end{gathered}
$$

with $i, j \in\{1,2\}$ and $i \neq j$. All of the terms have distributional limits which are at least $\mathcal{O}(\varepsilon)$. Simple cases which illustrate the estimate are:

$$
\begin{aligned}
I: & =\varepsilon \int d X d t\left[\left(\sqrt{\varepsilon} \int_{0}^{\frac{X}{\varepsilon}} \beta_{1}(s) d s\right) \frac{1}{\sqrt{\varepsilon}} \beta_{2}\left(\frac{X}{\varepsilon}\right)+\left(\sqrt{\varepsilon} \int_{0}^{\frac{X}{\varepsilon}} \beta_{2}(s) d s\right) \frac{1}{\sqrt{\varepsilon}} \beta_{1}\left(\frac{X}{\varepsilon}\right)\right] \varphi(X, X, t) \\
& =\frac{\varepsilon}{2} \int d X d t \partial_{X}\left(\sqrt{\varepsilon} \int_{0}^{\frac{X}{\varepsilon}} \beta_{1}(s) d s \sqrt{\varepsilon} \int_{0}^{\frac{X}{\varepsilon}} \beta_{2}(s) d s\right) \varphi(X, X, t) \\
& =-\frac{\varepsilon}{2} \int d X d t\left(\sqrt{\varepsilon} \int_{0}^{\frac{X}{\varepsilon}} \beta_{1}(s) d s \sqrt{\varepsilon} \int_{0}^{\frac{X}{\varepsilon}} \beta_{2}(s) d s\right) \partial_{X} \varphi(X, X, t) .
\end{aligned}
$$




$$
\begin{aligned}
I I: & =\varepsilon \int d X d t\left(\sqrt{\varepsilon} \int_{0}^{\frac{X}{\varepsilon}} \beta_{i}(s) d s\right) \frac{1}{\sqrt{\varepsilon}} \beta_{j}\left(\frac{X+\sqrt{g h} t}{\varepsilon}\right) \varphi(X+\sqrt{g h} t, X, t) \\
& =-\varepsilon \int d X d t\left(\sqrt{\varepsilon} \int_{0}^{\frac{X}{\varepsilon}} \beta_{i}(s) d s\right) \frac{1}{\sqrt{g h}} \partial_{t}\left(\sqrt{\varepsilon} \int_{0}^{\frac{X+\sqrt{g h t}}{\varepsilon}} \beta_{j}(s) d s\right) \varphi(X+\sqrt{g h} t, X, t) \\
& =-\frac{\varepsilon}{\sqrt{g h}} \int d X d t\left(\sqrt{\varepsilon} \int_{0}^{\frac{X}{\varepsilon}} \beta_{i}(s) d s\right)\left(\sqrt{\varepsilon} \int_{0}^{\frac{X+\sqrt{g h} t}{\varepsilon}} \beta_{j}(s) d s\right) \partial_{t} \varphi(X+\sqrt{g h} t, X, t) .
\end{aligned}
$$

In these expressions, notice that the factors that appear are continuous functionals on path space. Therefore, as $\varepsilon \mapsto 0$, they converge in law to functionals of Brownian motions [3]. The other remaining terms are easy to estimate.

3.3. Random characteristic coordinates. Our method to derive the long-wave limit gives rise to a version of the $\mathrm{KdV}$ equation which has coefficients which are realization dependent. That is, the approximation process does not fully homogenize, and there are persistent, realization dependent effects that are as important as the classical effects of dispersion and of nonlinear interactions. The principal manifestation of this is the random overall wavespeed, expressed in the limit as $\varepsilon \rightarrow 0$ as

$$
c_{0}(X, \omega)=\sqrt{g h}\left(1-\frac{\varepsilon^{3 / 2} \sigma_{\beta}}{2 h} \partial_{X} B(X)-\varepsilon^{2} a_{K d V}\right) .
$$

The constant $a_{K d V}$ is an adjustment to the characteristic velocity that is to be determined by an asymptotic analysis. The normally expected procedure is to solve the characteristic equations with this given wavespeed;

$$
\frac{d X}{d t}=c_{0}(X, \omega), \quad X(0)=Y
$$

to obtain characteristic coordinates $(Y, t)$ describing a net translational motion about which the more subtle nonlinear dispersive evolution takes place. In the context of a random bottom environment, however, the characteristic velocity field $c_{0}(X, \omega)$ in (3.39) has a component which is white noise, and when the flow of the characteristic vector field (3.40) is required, (3.39) is too singular to be able to make sense of a solution.

Our derivation of the $\mathrm{KdV}$ equation is nonetheless performed in characteristic coordinates. To do this, our alternative strategy is to use a natural regularization of the characteristic wavespeed given in (3.39) as an approximation, and to consider the characteristic coordinates indicated by (3.40) to be the limit as $\varepsilon \rightarrow 0$ of a sequence of more regular flows. The regularized characteristic vector field that we use is

$$
\begin{aligned}
& \frac{d X}{d t}=c_{\varepsilon}(X, \omega):=\sqrt{g h}\left(1-\frac{\varepsilon}{2 h} \beta\left(\frac{X}{\varepsilon}\right)-\varepsilon^{2} a_{K d V}\right) \\
& X(0)=Y .
\end{aligned}
$$

We remark that as long as $\beta(x, \omega) \in C^{1}(\mathbb{R})$ for P-a.e. realization $\omega$, the characteristic vector field $c_{\varepsilon}(X, \omega)$ is $C^{1}$, and for a given realization $\omega$ it is uniformly so in $\varepsilon$. Therefore the flows $X(t)=\Phi_{t}^{\varepsilon}(Y, \omega)$ exist for all $\varepsilon$, and lie in a bounded subset of $C^{1}$. The characteristics $X(t)$ are themselves $C^{1}$, and they are ordered by their initial values; if $Y_{1}<Y_{2}$ then for all $t, X_{1}=\Phi_{t}^{\varepsilon}\left(Y_{1}, \omega\right)<X_{2}=\Phi_{t}^{\varepsilon}\left(Y_{2}, \omega\right)$. As $\varepsilon \rightarrow 0$ there will normally not be a $C^{1}$ limit of the flows, but by standard compactness arguments there are limits $\Phi_{t}^{0}(Y, \omega)$ in any $C^{\alpha}(\mathbb{R}), 0 \leq \alpha<1$ which converge uniformly on compact sets, and which preserve the ordered property of 
the characteristics. Each such limit $X=\Phi_{t}^{0}(Y, \omega)$ can be taken to be a well-defined continuous and continuously invertible transformation.

To understand the asymptotic behavior of the transformation to characteristic coordinates, write

$$
\Phi_{t}^{\varepsilon}(Y, \omega)=X^{0}(t)+\varepsilon X^{1}(t)+\varepsilon^{2} X^{2}(t)+\ldots,
$$

where $X^{0}(0)=Y$, and $X^{j}(0)=0$ for $j \geq 1$ provide the initial conditions for the flow. Substituting this into the characteristic equation gives the result that

$$
\begin{aligned}
\frac{d X^{0}(t)}{d t} & =\sqrt{g h}, \quad X^{0}(t)=Y+t \sqrt{g h}, \\
\frac{d X^{1}(t)}{d t} & =-\frac{1}{2} \sqrt{\frac{g}{h}} \beta\left(X^{0}(t) / \varepsilon, \omega\right) \\
& =-\frac{1}{2} \sqrt{\frac{g}{h}} \beta((Y+t \sqrt{g h}) / \varepsilon, \omega),
\end{aligned}
$$

thus, variations to the characteristics are given by

$$
X^{1}(t)=-\frac{\varepsilon}{2 h} \int_{Y / \varepsilon}^{(Y+t \sqrt{g h}) / \varepsilon} \beta(s, \omega) d s .
$$

The final term relevant to our considerations is

$$
\frac{d X^{2}(t)}{d t}=-\sqrt{g h} a_{K d V}
$$

which integrates simply to $X^{2}(t)=-\sqrt{g h} a_{K d V} t$. Studying the integral expressions for $X^{1}(t)$ more closely, we find that

$$
X^{1}(t)=-\frac{\varepsilon}{2 h}\left(\int_{0}^{(Y+t \sqrt{g h}) / \varepsilon} \beta(s) d s-\int_{0}^{Y / \varepsilon} \beta(s) d s\right),
$$

which converges in law to Brownian motion as $\varepsilon \rightarrow 0$, according to our discussion in Section 3.2 Hence

$$
X^{1}(t)=-\frac{\sqrt{\varepsilon} \sigma_{\beta}}{2 h}(B(Y+t \sqrt{g h})-B(Y))+o(\sqrt{\varepsilon}) .
$$

In particular, the term $\varepsilon X^{1}(t)$ contributes at order $\varepsilon^{3 / 2}$. Due to Brownian scaling and to the property of independence of increments,

$$
X^{1}(t)=-\frac{\sqrt{\varepsilon} \sigma_{\beta}}{2 h} B_{\omega(Y)}(t \sqrt{g h})=-\sqrt{\varepsilon}\left(\frac{\sigma_{\beta}}{2 h} \sqrt[4]{g h}\right) B_{\omega(Y)}(t) .
$$

We note that the realizations $\omega(Y)$ of Brownian motion depend on the different initial positions $Y$, and in particular that for distinct initial points $Y_{1}$ and $Y_{2}$ the selection of realizations $B_{\omega\left(Y_{1}\right)}(t)$ and $B_{\omega\left(Y_{2}\right)}(t)$ of Brownian motion are independent, as long as $Y_{2}-Y_{1}>\sqrt{g h t}$. Putting this information together, an expression for the characteristic flow is given by

$$
\begin{aligned}
& X(t, Y ; \varepsilon, \omega)=Y+t \sqrt{g h}-\frac{\varepsilon^{2}}{2 h} \int_{Y / \varepsilon}^{(Y+t \sqrt{g h}) / \varepsilon} \beta(s, \omega) d s \\
& -\sqrt{g h} a_{K d V} \varepsilon^{2} t+\cdots .
\end{aligned}
$$


As $\varepsilon$ tends to 0 , the characteristics tend to the limiting distribution of paths given by

$$
X(t, Y ; \omega)=Y+t \sqrt{g h}-\frac{\varepsilon^{3 / 2} \sigma_{\beta}}{2 h} \sqrt[4]{g h} B_{\omega(Y)}(t)-\varepsilon^{2} \sqrt{g h} a_{K d V} t+\cdots .
$$

Inverting the expression gives a formula for $Y$ in terms of $X$ and $t$;

$$
\begin{aligned}
& Y(t, X ; \varepsilon, \omega)=\Phi_{-t}^{\varepsilon}(X ; \omega)=X-t \sqrt{g h}+\frac{\varepsilon^{2}}{2 h} \int_{(X-t \sqrt{g h}) / \varepsilon}^{X / \varepsilon} \beta(s, \omega) d s \\
& +\varepsilon^{2} \sqrt{g h} a_{K d V} t+\cdots .
\end{aligned}
$$

As $\varepsilon$ tends to 0 ,

$$
Y(t, X ; \omega)=X-t \sqrt{g h}+\frac{\varepsilon^{3 / 2} \sigma_{\beta}}{2 h} \sqrt[4]{g h} B_{\omega(X)}(t)+\sqrt{g h} a_{K d V} \varepsilon^{2} t+\cdots .
$$

The Jacobian of the flow has the following asymptotic expansion

$$
\frac{d X}{d Y}=1-\frac{\varepsilon}{2 h}\left[\beta\left(\frac{Y+\sqrt{g h t}}{\varepsilon}\right)-\beta\left(\frac{Y}{\varepsilon}\right)\right]=1+\mathcal{O}(\varepsilon) .
$$

In the limit as $\varepsilon$ tends to zero, the Jacobian (3.52), when multiplying a test function, behaves asymptotically as

$$
\frac{d X}{d Y} \sim 1-\frac{\varepsilon^{3 / 2} \sigma_{\beta}}{2 h} \sqrt[4]{g h} \partial_{X} B_{\omega(Y)}(t)
$$

\section{BoussinesQ REgime}

We now return to the expression (2.23) for the scaled Hamiltonian, in order to give a formal derivation of the appropriate Boussinesq system in this regime. Recall that $\beta$ is a mean zero, stationary mixing process with correlation function $\rho_{\beta}$. Using the analysis of the previous section, we write the leading order contributions of the second and fourth terms of (2.23) in the form

$$
\int_{-\infty}^{+\infty} \beta\left(\frac{X}{\varepsilon}, \omega\right)\left|D_{X} \xi(X)\right|^{2} d X=\sqrt{\varepsilon} \sigma_{\beta} \int \partial_{X} B(X)\left|D_{X} \xi(X)\right|^{2}+o(\sqrt{\varepsilon})
$$

and

$\int_{-\infty}^{+\infty}\left(\beta D_{x} \tanh \left(h D_{x}\right) \beta\right)\left(\frac{X}{\varepsilon}\right)\left|D_{X} \xi(X)\right|^{2} d X=\mathrm{E}\left(\beta D_{x} \tanh \left(h D_{x}\right) \beta\right) \int\left|D_{X} \xi(X)\right|^{2} d X$,

where as in (3.19) we calculate that

$$
\mathrm{E}\left(\beta D_{x} \tanh \left(h D_{x}\right) \beta\right)=\left(D_{y} \tanh \left(h D_{y}\right) \rho_{\beta}\right)(0):=a_{\beta} .
$$

The constant $a_{\beta}$ will contribute to an adjustment of the linear wavespeed in the Boussinesq regime. The equalities in (4.1) (4.2) are to be taken in the sense of law of the corresponding random processes.

We now include these expansions into the Hamiltonian, retaining terms up to order $\mathcal{O}\left(\varepsilon^{5}\right)$ and dropping those of higher order

$$
\begin{aligned}
H= & \frac{\varepsilon^{3}}{2} \int\left(\left(h-\varepsilon^{3 / 2} \sigma_{\beta} \partial_{X} B(X)-\varepsilon^{2} a_{\beta}\right)\left|D_{X} \xi\right|^{2}+g \eta^{2}\right) d X \\
& +\frac{\varepsilon^{5}}{2} \int\left(\xi D_{X} \eta D_{X} \xi-\frac{h^{3}}{3} \xi D_{X}^{4} \xi\right) d X+o\left(\varepsilon^{5}\right) .
\end{aligned}
$$


We note the rôle of a stochastic effective depth played by

$$
h_{0}(X)=h-\varepsilon^{3 / 2} \sigma_{\beta} \partial_{X} B(X)-\varepsilon^{2} a_{\beta}+o\left(\varepsilon^{2}\right)
$$

which is a function of the long length scale variables alone. Since it is normally not necessary to introduce characteristic coordinates in this derivation, a regularization such as described in section 3.3 is not required, and the limiting effective depth $h_{0}(X)$ is used directly in the averaged Hamiltonian.

Changing the variables $(\eta, \xi)$ to $\left(\eta, u=\partial_{X} \xi\right)$, the Hamiltonian becomes

$$
H_{1}=\frac{\varepsilon^{3}}{2} \int\left(h_{0}(X) u^{2}+g \eta^{2}-\varepsilon^{2}\left(\frac{h^{3}}{3}\left(\partial_{X} u\right)^{2}-\eta u^{2}\right)\right) d X .
$$

The symplectic structure has to be modified accordingly as in 8 . Consider the transformation $w \rightarrow v=f(w)$, which transforms Hamilton's equations

$$
\partial_{t} w=J \delta_{w} H(w)
$$

to the form

$$
\partial_{t} v=J_{1} \delta_{v} H_{1}(v)
$$

with a new symplectic structure

$$
J_{1}=\partial_{w} f J\left(\partial_{w} f\right)^{\top},
$$

where $\partial_{w} f$ is the Jacobian of the map $f$. In our case, $w=\left(\begin{array}{l}\eta \\ \xi\end{array}\right), \quad v=\left(\begin{array}{l}\eta \\ u\end{array}\right)=$ $\left(\begin{array}{cc}I & 0 \\ 0 & \partial_{X}\end{array}\right) w$, and the matrix $J=\varepsilon^{-3}\left(\begin{array}{cc}0 & I \\ -I & 0\end{array}\right)$ is transformed to $J_{1}=\varepsilon^{-3}\left(\begin{array}{cc}0 & -\partial_{X} \\ -\partial_{X} & 0\end{array}\right)$, where the power of $\varepsilon$ is due to the scaling transformations (2.15). The evolution equations take the form

$$
\partial_{t}\left(\begin{array}{l}
\eta \\
u
\end{array}\right)=J_{1}\left(\begin{array}{l}
\delta_{\eta} H_{1} \\
\delta_{u} H_{1}
\end{array}\right) .
$$

In the end we find the Boussinesq system in the form

$$
\begin{aligned}
\partial_{t} \eta & =-\partial_{X}\left(\left(h_{0}(X)+\varepsilon^{2} \eta\right) u\right)-\varepsilon^{2} \frac{h^{3}}{3} \partial_{X}^{3} u, \\
\partial_{t} u & =-g \partial_{X} \eta-\varepsilon^{2} u \partial_{X} u .
\end{aligned}
$$

While this form of Boussinesq system appears most naturally from a direct expansion of the Hamiltonian of the problem of water waves, the resulting system of partial differential equations is not well posed, and it is rarely used directly in modeling. In the present setting, the situation is further aggravated by the fact that a coefficient in the above system is singular, as it involves the second derivative of a Brownian motion. Several routes to resolving these issues are possible, modifying the linear dispersion relation for the Boussinesq system, and regularizing the coefficients as in section 3.3, for example. However we will not pursue this direction of inquiry in the present paper, preferring to make a more systematic study of the $\mathrm{KdV}$ scaling regime. 


\section{The KDV REGIME}

In the case of the Boussinesq derivation, the limit of certain integrals in the water waves Hamiltonian will give rise to singular coefficients in the resulting equations of motion. This is even more true in the case of the $\mathrm{KdV}$ regime; indeed the transformation to characteristic coordinates will give rise to a modified symplectic structure which involves a second derivative of Brownian motion, something that is not acceptable on an analytic level. To get around this difficulty, we regularize the linear wavespeed as described in section 3.3. a process which consists of retaining certain terms with rapidly varying coefficients in the Hamiltonian, and only taking the limit after the long wave equations are derived. We assume that $\sigma_{\beta}>0$, which implies that the resulting realization dependent fluctuations are maximally significant in the limit, and we will perform the smoothing procedure in a way which is consistent with this assumption.

5.1. Successive changes of variables. We start again from the expression (2.23) for the Hamiltonian. As in the derivation of the Boussinesq system, we first change the variables $(\eta, \xi)$ to $\left(\eta, u=\partial_{X} \xi\right)$, leading to a transformed Hamiltonian $H_{1}^{\varepsilon}$ defined by

$$
H_{1}^{\varepsilon}=\frac{\varepsilon^{3}}{2} \int\left(h_{\varepsilon}(X) u^{2}+g \eta^{2}-\varepsilon^{2}\left(\frac{h^{3}}{3}\left(\partial_{X} u\right)^{2}-\eta u^{2}\right)\right) d X
$$

and a modified symplectic structure $J_{1}=\varepsilon^{-3}\left(\begin{array}{cc}0 & -\partial_{X} \\ -\partial_{X} & 0\end{array}\right)$. The next change of variables is defined by the transformation

$$
\eta=\sqrt[4]{\frac{h_{\varepsilon}}{4 g}}(r+s), \quad u=\sqrt[4]{\frac{g}{4 h_{\varepsilon}}}(r-s) .
$$

The new symplectic structure resulting from this transformation is

$$
J_{2}=\varepsilon^{-3}\left(\begin{array}{cc}
-\partial_{X} & \frac{1}{4} \frac{\partial_{X} h_{\varepsilon}}{h_{\varepsilon}} \\
-\frac{1}{4} \frac{\partial_{X} h_{\varepsilon}}{h_{\varepsilon}} & \partial_{X}
\end{array}\right),
$$

whose off-diagonal terms quantify the scattering of solutions due to variations in the topography. In this expression, we retain the regularized expression :

$$
h_{\varepsilon}(X)=h-\varepsilon \beta\left(\frac{X}{\varepsilon}\right)-\varepsilon^{2} a_{\beta} .
$$

The Hamiltonian is written as

$$
\begin{gathered}
H_{2}^{\varepsilon}(r, s)=\frac{\varepsilon^{3}}{2} \int\left(\sqrt{g h_{\varepsilon}(X)}\left(r^{2}+s^{2}\right)-\frac{\varepsilon^{2}}{3} h^{3}\left[\left(\partial_{X} \sqrt[4]{\frac{g}{4 h_{\varepsilon}}} r\right)^{2}\right.\right. \\
\left.-2\left(\partial_{X} \sqrt[4]{\frac{g}{4 h_{\varepsilon}}} r\right)\left(\partial_{X} \sqrt[4]{\frac{g}{4 h_{\varepsilon}}} s\right)+\left(\partial_{X} \sqrt[4]{\frac{g}{4 h_{\varepsilon}}} s\right)^{2}\right] \\
\left.+\frac{\varepsilon^{2}}{2} \sqrt[4]{\frac{g}{4 h_{\varepsilon}}}\left(r^{3}-r^{2} s-r s^{2}+s^{3}\right)\right) d X+o\left(\varepsilon^{5}\right) .
\end{gathered}
$$

Notice that, except for the first term in the Hamiltonian, $h_{\varepsilon}$ appears in terms that are already of order $\varepsilon^{5}$ and thus can be replaced there by the constant $h$ in this 
asymptotic calculation. Denoting by

$$
c_{1}=\frac{h^{3}}{3} \sqrt{\frac{g}{4 h}}, \quad c_{2}=\frac{1}{2} \sqrt[4]{\frac{g}{4 h}}
$$

we rewrite $H_{2}^{\varepsilon}$ in the form:

$$
\begin{aligned}
H_{2}^{\varepsilon} & =\frac{\varepsilon^{3}}{2} \int \sqrt{g h_{\varepsilon}}\left(r^{2}+s^{2}\right)-c_{1} \varepsilon^{2}\left(\left(\partial_{X} r\right)^{2}-2\left(\partial_{X} r\right)\left(\partial_{X} s\right)+\left(\partial_{X} s\right)^{2}\right) \\
& +c_{2} \varepsilon^{2}\left(r^{3}-r^{2} s-r s^{2}+s^{3}\right) d X+o\left(\varepsilon^{5}\right) .
\end{aligned}
$$

Hamilton's equations for $(r, s)$ take the form

$$
\partial_{t}\left(\begin{array}{l}
r \\
s
\end{array}\right)=J_{2}\left(\begin{array}{c}
\delta_{r} H_{2}^{\varepsilon} \\
\delta_{s} H_{2}^{\varepsilon}
\end{array}\right)
$$

where $\delta_{r} H_{2}^{\varepsilon}$ and $\delta_{s} H_{2}^{\varepsilon}$ are computed as follows:

$$
\begin{aligned}
& \delta_{r} H_{2}^{\varepsilon}=\frac{\varepsilon^{3}}{2}\left(\sqrt{g h_{\varepsilon}} 2 r+c_{1} \varepsilon^{2}\left(2 \partial_{X}^{2} r-2 \partial_{X}^{2} s\right)+c_{2} \varepsilon^{2}\left(3 r^{2}-2 r s-s^{2}\right)\right) \\
& \delta_{s} H_{2}^{\varepsilon}=\frac{\varepsilon^{3}}{2}\left(\sqrt{g h_{\varepsilon}} 2 s-c_{1} \varepsilon^{2}\left(2 \partial_{X}^{2} r-2 \partial_{X}^{2} s\right)-c_{2} \varepsilon^{2}\left(r^{2}+2 r s-3 s^{2}\right)\right) .
\end{aligned}
$$

Hamilton's equations are explicitly

$$
\begin{aligned}
\partial_{t} r= & -\partial_{X}\left[\sqrt{g h_{\varepsilon}} r+\varepsilon^{2}\left(c_{1}\left(\partial_{X}^{2} r-\partial_{X}^{2} s\right)+\frac{1}{2} c_{2}\left(3 r^{2}-2 r s-s^{2}\right)\right)\right] \\
& +\frac{1}{4} \frac{\partial_{X} h_{\varepsilon}}{h_{\varepsilon}}\left[\sqrt{g h_{\varepsilon}} s+\varepsilon^{2}\left(c_{1}\left(\partial_{X}^{2} s-\partial_{X}^{2} r\right)+\frac{1}{2} c_{2}\left(-r^{2}-2 r s+3 s^{2}\right)\right)\right] \\
\partial_{t} s= & \partial_{X}\left[\sqrt{g h_{\varepsilon}} s+\varepsilon^{2}\left(c_{1}\left(\partial_{X}^{2} s-\partial_{X}^{2} r\right)+\frac{1}{2} c_{2}\left(-r^{2}-2 r s+3 s^{2}\right)\right)\right] \\
& -\frac{1}{4} \frac{\partial h_{\varepsilon}}{h_{\varepsilon}}\left[\sqrt{g h_{\varepsilon}} r+\varepsilon^{2}\left(c_{1}\left(\partial_{X}^{2} r-\partial_{X}^{2} s\right)+\frac{1}{2} c_{2}\left(3 r^{2}-2 r s-s^{2}\right)\right)\right] .
\end{aligned}
$$

In the action of $J_{2} \delta H_{2}^{\varepsilon}$, there are products of $h_{\varepsilon}$ and its derivatives, and each factor tends to a distribution (see Lemma 3.2) in the limit $\varepsilon \rightarrow 0$. The product is nevertheless well defined because of the form it takes:

$$
\int \partial_{X} h_{\varepsilon} h_{\varepsilon}{ }^{-1 / 2} f(X) d X=2 \int \partial_{X} h_{\varepsilon}^{1 / 2} f(X) d X
$$

We perform an additional change of scale of $s$ relative to $r$ defined by

$$
\left(\begin{array}{c}
r \\
s_{1}
\end{array}\right)=\left(\begin{array}{cc}
1 & 0 \\
0 & \varepsilon^{-3 / 2}
\end{array}\right)\left(\begin{array}{l}
r \\
s
\end{array}\right)
$$

which puts forward $r(X, t)$ as the main component of the solution which is anticipated to be traveling principally to the right, with a relatively small scattered component $s_{1}(X, t)$ propagating principally to the left. The transformation leads to a modified symplectic structure

$$
J_{3}=\frac{1}{\varepsilon^{3}}\left(\begin{array}{cc}
-\partial_{X} & \frac{1}{4 \varepsilon^{3 / 2}} \frac{\partial_{X} h_{\varepsilon}}{h_{\varepsilon}} \\
-\frac{1}{4 \varepsilon^{3 / 2}} \frac{\partial_{X} h_{\varepsilon}}{h_{\varepsilon}} & \frac{1}{\varepsilon^{3}} \partial_{X}
\end{array}\right)
$$


and a final Hamiltonian

$$
\begin{aligned}
H_{3}^{\varepsilon}\left(r, s_{1}\right) & =\frac{\varepsilon^{3}}{2} \int \sqrt{g h_{\varepsilon}}\left(r^{2}+\varepsilon^{3} s_{1}^{2}\right)-c_{1} \varepsilon^{2}\left(\left(\partial_{X} r\right)^{2}-2 \varepsilon^{\frac{3}{2}}\left(\partial_{X} r\right)\left(\partial_{X} s_{1}\right)+\varepsilon^{3}\left(\partial_{X} s_{1}\right)^{2}\right) \\
& +c_{2} \varepsilon^{2}\left(r^{3}-\varepsilon^{\frac{3}{2}} r^{2} s_{1}-\varepsilon^{3} r s_{1}^{2}+\varepsilon^{\frac{9}{2}} s_{1}^{3}\right) d X+o\left(\varepsilon^{5}\right) .
\end{aligned}
$$

The equations stemming from the Hamiltonian (5.13) and the above symplectic structure are

$$
\begin{aligned}
& \partial_{t} r=- \partial_{X}\left[\sqrt{g h_{\varepsilon}} r+\varepsilon^{2}\left(c_{1} \partial_{X}^{2} r+\frac{3}{2} c_{2} r^{2}\right)\right. \\
&\left.+\varepsilon^{2}\left(-\varepsilon^{\frac{3}{2}} c_{1} \partial_{X}^{2} s_{1}-\varepsilon^{\frac{3}{2}} c_{2} r s_{1}-\frac{1}{2} \varepsilon^{3} c_{2} s_{1}^{2}\right)\right] \\
&-\frac{1}{4} \frac{\partial_{x} \beta\left(\frac{X}{\varepsilon}\right)}{h_{\varepsilon}}\left[\varepsilon^{3 / 2} \sqrt{g h_{\varepsilon}} s_{1}+\varepsilon^{2}\left(c_{1}\left(-\partial_{X}^{2} r+\varepsilon^{3 / 2} \partial_{X}^{2} s_{1}\right)\right.\right. \\
&\left.\left.+c_{2}\left(-\frac{1}{2} r^{2}-\varepsilon^{3 / 2} r s_{1}+\frac{3}{2} \varepsilon^{3} s_{1}^{2}\right)\right)\right] \\
& \partial_{t} s_{1}=\partial_{X}\left[\sqrt{g h_{\varepsilon}} s_{1}+\varepsilon^{2}\left(c_{1}\left(-\varepsilon^{-\frac{3}{2}} \partial_{X}^{2} r+\partial_{X}^{2} s_{1}\right)\right.\right. \\
&\left.\left.+c_{2}\left(-\frac{1}{2} \varepsilon^{-\frac{3}{2}} r^{2}-r s_{1}+\frac{3}{2} \varepsilon^{\frac{3}{2}} s_{1}^{2}\right)\right)\right] \\
&+\frac{1}{4} \frac{\partial_{x} \beta\left(\frac{X}{\varepsilon}\right)}{h_{\varepsilon}}\left[\varepsilon^{-\frac{3}{2}} \sqrt{g h_{\varepsilon}} r+\varepsilon^{\frac{1}{2}}\left(c_{1}\left(\partial_{X}^{2} r-\varepsilon^{\frac{3}{2}} \partial_{X}^{2} s_{1}\right)\right.\right. \\
&\left.\left.+c_{2}\left(\frac{3}{2} r^{2}-\varepsilon^{\frac{3}{2}} r s_{1}-\frac{1}{2} \varepsilon^{3} s_{1}^{2}\right)\right)\right] .
\end{aligned}
$$

It is ambiguous at this point precisely which terms of the above system of partial differential equations play a rôle in the asymptotic description of solutions in the limit as $\varepsilon$ tends to zero. The transformation (5.11) is not homogeneous in the perturbation parameter $\varepsilon$, and because of fluctuations there are numerous cancellations that occur in the remaining terms, not all of them having an influence on the asymptotic regime (see Lemmas 3.2 and 3.5 for example). We will show in the subsequent analysis of Section 5.3 that the asymptotic behavior of solutions of equations (5.14)(5.15) as $\varepsilon \rightarrow 0$ is governed by the following coupled system of equations, with an appropriate choice of the parameters $a_{K d V}$ and $b$.

$$
\begin{gathered}
\partial_{t} r=-\partial_{X}\left[c_{\varepsilon}(X) r+\varepsilon^{2}\left(c_{1} \partial_{X}^{2} r+\frac{3}{2} c_{2} r^{2}\right)\right]+\varepsilon^{2} b r \\
\partial_{t} s_{1}=\sqrt{g h} \partial_{X} s_{1}+\frac{1}{4} \sqrt{\frac{g}{h}} \varepsilon^{-3 / 2} \partial_{x} \beta\left(\frac{X}{\varepsilon}\right) r,
\end{gathered}
$$

where the regularized velocity is $c_{\varepsilon}(X)=\sqrt{g h}\left(1-\frac{\varepsilon}{2 h} \beta(X / \varepsilon)-\varepsilon^{2} a_{K d V}\right)$. There are two free parameters in this system of equations, namely, $a_{K d V}$ and $b$. They will be determined by the consistency analysis of Section 5.3 as fixed points of the solution process and the asymptotic analysis. In the end we find that

$$
\begin{aligned}
& a_{K d V}=\frac{1}{2 h} a_{\beta}+\frac{1}{4 h^{2}} \mathrm{E}\left(\beta^{2}\right)+\frac{3 c_{1}}{8 h^{2} \sqrt{g h}} \mathrm{E}\left(\left(\partial_{x} \beta\right)^{2}\right) \\
& b=-\frac{7 c_{1}}{64 h^{3}} \mathrm{E}\left(\left(\partial_{x} \beta\right)^{3}\right) .
\end{aligned}
$$


5.2. Solution procedure for the random $\mathbf{K d V}$ equations. In this section we describe a reduction procedure for the system of equations (5.16)-(5.17) that expresses the solution component $r(X, t)$ in terms of a solution $q(Y, \tau)$ of a deterministic equation similar to the $\mathrm{KdV}$ equation, under a random change of variables $(Y \mapsto X(t, Y))$ and a scaling $\tau=\varepsilon^{2} t$ to the $\mathrm{KdV}$ time. The scattered component $s_{1}(X, t)$ is an expression involving integrations along characteristics. The solution depends upon the two parameters $a_{K d V}$ and $b$. We retain the regularized form of the characteristic velocity $c_{\varepsilon}(X)$, only taking the limit as $\varepsilon \rightarrow 0$ in expressions for the solution.

Substitute $r=\partial_{X} R$ into (5.16); the resulting equation for $R$ is

$$
\partial_{t} R=-c_{\varepsilon}(X) \partial_{X} R-\varepsilon^{2}\left(c_{1} \partial_{X}^{3} R+\frac{3}{2} c_{2}\left(\partial_{X} R\right)^{2}\right)+\varepsilon^{2} b R .
$$

Transform to characteristic coordinates as in Section 3.3

$$
\frac{d X}{d t}=c_{\varepsilon}(X), \quad X(0)=Y
$$

We denote the flow by $X=\Phi_{t}^{\varepsilon}(Y)$, which is a regularized realization dependent change of variables. Define $Q(Y, \tau)=R(X, t)$ so that $Q$ satisfies

$$
\partial_{\tau} Q=-c_{1} \partial_{Y}^{3} Q-\frac{3}{2} c_{2}\left(\partial_{Y} Q\right)^{2}+b Q
$$

To solve the initial value problem, set $q(Y, 0)=r(Y, 0)=r^{0}(Y)$, and solve the deterministic equation

$$
\partial_{\tau} q=-c_{1} \partial_{Y}^{3} q-3 c_{2} q \partial_{Y} q+b q
$$

for $q(Y, \tau)=\partial_{Y} Q(Y, \tau)$. If $b=0$, equation (5.23) is the classical KdV equation. Additionally, for each realization $\beta(x, \omega)$ the regularized ODE (5.21) defining the flow has a solution given by $X=X(t, Y ; \varepsilon, \omega)$. With these two ingredients, the solution $r(X, t)$ of equation (5.16) is given by

$$
r(X, t)=\partial_{X} Q\left(Y(t, X ; \varepsilon, \omega), \varepsilon^{2} t\right)=\partial_{Y} Q\left(Y(t, X ; \varepsilon, \omega), \varepsilon^{2} t\right) \partial_{X} Y(t, X ; \varepsilon, \omega)
$$

where $\partial_{Y} X(t, Y ; \varepsilon, \omega)$ is the Jacobian of the flow (5.21) as described in section 3.3. and $\partial_{X} Y(t, X ; \varepsilon, \omega)$ is its inverse. This is an expression of the solution of the regularized equation.

The equation (5.17) describes the scattered component of the KdV system above, whose solution is expressed by integration of a forcing term which is given in terms of $r(X, t)$ along left-moving characteristics . Explicitly,

$$
\begin{aligned}
s_{1}(X, t)= & s_{1}^{0}(X+\sqrt{g h} t) \\
& +\frac{\varepsilon^{-\frac{3}{2}}}{4} \sqrt{\frac{g}{h}} \int_{0}^{t} \partial_{x} \beta\left(\frac{X+\sqrt{g h}\left(t-t^{\prime}\right)}{\varepsilon}\right) r\left(X+\sqrt{g h}\left(t-t^{\prime}\right), t^{\prime}\right) d t^{\prime} \\
= & s_{1}^{0}(X+\sqrt{g h} t)+\frac{\varepsilon^{-\frac{3}{2}}}{4 h} \int_{X}^{X+\sqrt{g h} t} \partial_{x} \beta\left(\frac{\theta}{\varepsilon}\right) r\left(\theta, t+\frac{X-\theta}{\sqrt{g h}}\right) d \theta .
\end{aligned}
$$

The small parameter $\varepsilon$ is still present in the regularization; to complete the description we consider the limit of the expressions (5.24) (5.25) as $\varepsilon$ tends to zero. The solution of (5.22) is smooth, and admits a Taylor expansion in its arguments. The 
inverse Jacobian has an asymptotic expression as well. Therefore, one writes

$$
\begin{aligned}
r(X, t)= & \partial_{X} Q\left(Y(X, t ; \omega), \varepsilon^{2} t\right)=\partial_{Y} Q(Y(X, t ; \omega), t) \partial_{X} Y(X, t ; \omega) \\
= & q\left(X-\sqrt{g h} t, \varepsilon^{2} t\right)\left(1+\frac{\varepsilon}{2 h}\left(\beta\left(\frac{X}{\varepsilon}\right)-\beta\left(\frac{X-\sqrt{g h} t}{\varepsilon}\right)\right)\right. \\
& +\partial_{X} q\left(X-\sqrt{g h} t, \varepsilon^{2} t\right) \frac{\varepsilon^{2}}{2 h} \int_{(X-\sqrt{g h} t) / \varepsilon}^{\frac{X}{\varepsilon}} \beta\left(t^{\prime}\right) d t^{\prime}+\cdots \\
=\quad & q\left(X-\sqrt{g h} t, \varepsilon^{2} t\right) \\
& +\partial_{X}\left(q\left(X-\sqrt{g h} t, \varepsilon^{2} t\right)\left(\frac{\varepsilon^{2}}{2 h} \int_{(X-\sqrt{g h} t) / \varepsilon}^{\frac{X}{\varepsilon}} \beta\left(t^{\prime}\right) d t^{\prime}\right)\right)+\mathcal{O}\left(\varepsilon^{2}\right) .
\end{aligned}
$$

Proposition 5.1. In the limit as $\varepsilon$ tends to zero, the expression (5.26) for the solution of (5.16) is asymptotic as a distribution to

$$
\begin{aligned}
r(X, t)= & q\left(X-\sqrt{g h} t, \varepsilon^{2} t\right) \\
& +\frac{\varepsilon^{3 / 2} \sigma_{\beta}}{2 h} \sqrt[4]{g h} \partial_{X}\left(q\left(X-\sqrt{g h} t, \varepsilon^{2} t\right) B_{\omega(X)}(t)\right)+o\left(\varepsilon^{3 / 2}\right) .
\end{aligned}
$$

The expression for (5.25) for the solution $s_{1}$ is asymptotic as a distribution to

$$
\begin{aligned}
& s_{1}(X, t)=s_{1}^{0}(X+\sqrt{g h} t) \\
& +\frac{1}{4 h \sigma_{\beta}} \int_{X}^{X+\sqrt{g h} t} B(\theta) \frac{d^{2}}{d \theta^{2}} q\left(2 \theta-X-\sqrt{g h} t, \varepsilon^{2}\left(t+\frac{X-\theta}{\sqrt{g h}}\right)\right) d \theta \\
& +\frac{1}{4 h \sigma_{\beta}}\left(\partial_{X} B(X+\sqrt{g h} t) q(X+\sqrt{g h} t, 0)-\partial_{X} B(X) q\left(X-\sqrt{g h} t, \varepsilon^{2} t\right)\right) \\
& -\frac{1}{2 h \sigma_{\beta}}\left(B(X+\sqrt{g h} t) \partial_{X} q(X+\sqrt{g h} t, 0)-B(X) \partial_{X} q\left(X-\sqrt{g h} t, \varepsilon^{2} t\right)\right) .
\end{aligned}
$$

Proof. The expression for the limit of $r_{1}$ follows directly from the application of Lemma 3.5. It is an expression which exhibits both randomness in its amplitude, as well as in location as per the random characteristic coordinates in which it is expressed. For the calculation for the limit of $s_{1}$, we substitute the expression (5.26) in 5.25 :

$$
\begin{aligned}
& s_{1}(X, t)=s_{1}^{0}(X+\sqrt{g h} t) \\
& +\frac{\varepsilon^{-\frac{3}{2}}}{4 h} \int_{X}^{X+\sqrt{g h t}} \partial_{x} \beta\left(\frac{\theta}{\varepsilon}\right) q\left(2 \theta-X-\sqrt{g h} t, \varepsilon^{2}\left(t+\frac{X-\theta}{\sqrt{g h}}\right)\right) d \theta \\
& +\frac{\varepsilon^{1 / 2}}{8 h^{2}} \int_{X}^{X+\sqrt{g h t} t} \partial_{x} \beta\left(\frac{\theta}{\varepsilon}\right)\left[\int_{\frac{2 \theta-X-\sqrt{g h t}}{\varepsilon}}^{\frac{\theta}{\varepsilon}} \beta(s) d s\right. \\
& \left.\quad \times \partial_{X} q\left(2 \theta-X-\sqrt{g h} t, \varepsilon^{2}\left(t+\frac{X-\theta}{\sqrt{g h}}\right)\right)\right] d \theta \\
& +\frac{\varepsilon^{-\frac{1}{2}}}{8 h^{2}} \int_{X}^{X+\sqrt{g h t}} \quad\left(\partial_{x} \frac{\beta^{2}}{2}\left(\frac{\theta}{\varepsilon}\right)-\partial_{x} \beta\left(\frac{\theta}{\varepsilon}\right) \beta\left(\frac{2 \theta-X-\sqrt{g h} t}{\varepsilon}\right)\right) \\
& \quad \times q\left(2 \theta-X-\sqrt{g h} t, \varepsilon^{2}\left(t+\frac{X-\theta}{\sqrt{g h}}\right)\right) d \theta .
\end{aligned}
$$

Except for the first term $s_{1}^{0}$ that remains unchanged, all the terms appearing in the limiting expression (5.28) come from the first integral in the expression of $s_{1}$, where we performed several integrations by parts and use the fact that $\partial_{t} q\left(X, \varepsilon^{2} t\right)$ 
is $\mathcal{O}\left(\varepsilon^{2}\right)$. By more integration by parts, using the fact that $\partial_{x}=\varepsilon \partial_{X}$ we can show that the third term (third and fourth lines) in the expression (5.29) is $\mathcal{O}\left(\varepsilon^{1 / 2}\right)$. Let us turn to the last term (fifth and sixth lines) of (5.29). For the term containing $\partial_{x} \beta^{2}(\theta / \varepsilon)$, integration by parts will produce an additional $\varepsilon$ and the term will eventually be of order $\mathcal{O}\left(\varepsilon^{1 / 2}\right)$. To estimate the term containing the product $\partial_{x} \beta\left(\frac{\theta}{\varepsilon}\right) \beta\left(\frac{2 \theta-X-\sqrt{g h} t}{\varepsilon}\right)$, the integration by parts moves the derivative $\partial_{x}$ to all other terms. The only contribution that will not produce an $\varepsilon$ is when the derivative acts on $\beta\left(\frac{2 \theta-X-\sqrt{g h t}}{\varepsilon}\right)$. For this term, we write

$$
\partial_{x} \beta\left(\frac{2 \theta-X-\sqrt{g h} t}{\varepsilon}\right)=-\frac{\varepsilon}{\sqrt{g h}} \partial_{t} \beta\left(\frac{2 \theta-X-\sqrt{g h} t}{\varepsilon}\right) .
$$

After some simple manipulations, this term is again $\mathcal{O}\left(\varepsilon^{1 / 2}\right)$.

5.3. Consistency of the resulting system of equations. In this subsection, we complete the cycle of a self-consistency analysis for equations (5.16) and (5.17), out of which the two so-far undetermined constants $a_{K d V}$ and $b$ are selected. It is clear that not all terms in equations (5.14) and (5.15) are of equal importance in the limit as $\varepsilon \rightarrow 0$. Recall the criterion as presented in section 3, which states that a term $a(X, t ; \varepsilon, \omega)$ is of order $\mathcal{O}\left(\varepsilon^{r}\right)$ if for any space-time test function $\varphi(X, t) \in \mathcal{S}$ the measures $\mathrm{P}_{\varepsilon}$ induced by $\varepsilon^{-r} \int a(X, t ; \varepsilon, \omega) \varphi(X, t) d X d t$ converge weakly to a limit $\mathrm{P}_{0}$ as $\varepsilon$ tends to zero. In the present case, the analysis consists of (i) the derivation of an expression for the solutions of (5.16) (5.17) which are stated in (5.25) and (5.26), and depend upon the two parameters $a_{K d V}$ and $b$; (ii) the examination of the terms in (5.14), including in particular those which do not appear in (5.16) (respectively, all the terms in (5.15), in particular those that do not appear in (5.17)). Using the expressions (5.25) (5.26) we then show that, except terms which appear in (5.16) (respectively (5.17) ), they are asymptotically of order $o\left(\varepsilon^{2}\right)$ (respectively, of order $o(1)$ ). Both the system (5.16) (5.17) and the solution expressions (5.25) (5.26) depend upon parameters $a_{K d V}$ and $b$. (iii) The demonstration that these constants can be chosen so that there is a fixed point of this analysis. Namely, the solution depending upon the constants $a_{K d V}$ and $b$ has asymptotic behavior which satisfies the equations (5.16)-(5.17) with the same choice of constants.

Let us denote the terms in (5.14) by

$$
\begin{aligned}
& \mathrm{I}_{r}=\varepsilon^{2} \partial_{X}\left(-\varepsilon^{3 / 2} c_{1} \partial_{X}^{2} s_{1}-\varepsilon^{3 / 2} c_{2} r s_{1}-\frac{1}{2} \varepsilon^{3} c_{2} s_{1}^{2}\right) \\
& \mathrm{II}_{r}=-\frac{1}{4} \frac{\partial_{x} \beta\left(\frac{X}{\varepsilon}\right)}{h_{\varepsilon}} \varepsilon^{3 / 2} \sqrt{g h_{\varepsilon}} s_{1} \\
& \mathrm{III}_{r}=-\frac{1}{4} \frac{\partial_{x} \beta\left(\frac{X}{\varepsilon}\right)}{h_{\varepsilon}} \varepsilon^{2} c_{1}\left(-\partial_{X}^{2} r+\varepsilon^{3 / 2} \partial_{X}^{2} s_{1}\right) \\
& \mathrm{IV}_{r}=-\frac{1}{4} \frac{\partial_{x} \beta\left(\frac{X}{\varepsilon}\right)}{h_{\varepsilon}} \varepsilon^{2} c_{2}\left(-\frac{1}{2} r^{2}-\varepsilon^{3 / 2} r s_{1}+\frac{3}{2} \varepsilon^{3} s_{1}^{2}\right) .
\end{aligned}
$$

Similarly, we denote the terms in (5.15) by 


$$
\begin{aligned}
& \mathrm{I}_{s}=\varepsilon^{2} \partial_{X}\left(c_{1}\left(-\varepsilon^{-3 / 2} \partial_{X}^{2} r+\partial_{X}^{2} s_{1}\right)+c_{2}\left(-\frac{1}{2} \varepsilon^{-3 / 2} r^{2}-r s_{1}+\frac{3}{2} \varepsilon^{3 / 2} s_{1}^{2}\right)\right) \\
& \mathrm{II}_{s}=\frac{1}{4} \frac{\partial_{x} \beta\left(\frac{X}{\varepsilon}\right)}{h_{\varepsilon}} \varepsilon^{-3 / 2} \sqrt{g h_{\varepsilon}} r \\
& \mathrm{III}_{s}=\frac{1}{4} \frac{\partial_{x} \beta\left(\frac{X}{\varepsilon}\right)}{h_{\varepsilon}} \varepsilon^{1 / 2} c_{1}\left(\partial_{X}^{2} r-\varepsilon^{3 / 2} \partial_{X}^{2} s_{1}\right) \\
& \mathrm{IV}_{s}=\frac{1}{4} \frac{\partial_{x} \beta\left(\frac{X}{\varepsilon}\right)}{h_{\varepsilon}} \varepsilon^{1 / 2} c_{2}\left(\frac{3}{2} r^{2}-\varepsilon^{3 / 2} r s_{1}-\frac{1}{2} \varepsilon^{3} s_{1}^{2}\right) .
\end{aligned}
$$

The purpose is to evaluate the asymptotic behavior of each of these terms as $\varepsilon \rightarrow 0$.

Lemma 5.2. The term $\mathrm{II}_{r}$ has the asymptotic behavior

$$
\mathrm{II}_{r}=\frac{1}{8 h} \sqrt{\frac{g}{h}} \varepsilon^{2} \mathrm{E}\left(\beta^{2}\right) \partial_{X} r(X, t)+o\left(\varepsilon^{2}\right) .
$$

Lemma 5.3. The term $\mathrm{II}_{s}$ has the behavior

$$
\mathrm{II}_{s}=\frac{\varepsilon^{-3 / 2}}{4} \sqrt{\frac{g}{h}} \partial_{x} \beta\left(\frac{X}{\varepsilon}\right) r+o(1)
$$

and this expression has an asymptotic limit as $\varepsilon \rightarrow 0$ which is

$$
\frac{1}{4} \sqrt{\frac{g}{h}} \sigma_{\beta} \partial_{X}^{2} B(X, \omega) q(X-\sqrt{g h} t, \tau)
$$

Lemma 5.4.

$$
\begin{gathered}
\mathrm{III}_{r}=\frac{3 c_{1}}{8 h^{2}} \varepsilon^{2} \mathrm{E}\left(\left(\partial_{x} \beta\right)^{2}\right) \partial_{X} r-\frac{7 c_{1}}{64 h^{3}} \varepsilon^{2} \mathrm{E}\left(\left(\partial_{x} \beta\right)^{3}\right) r+o\left(\varepsilon^{2}\right), \\
\mathrm{III}_{s}=\varepsilon^{-3 / 2} \mathrm{III}_{r}=\mathcal{O}\left(\varepsilon^{1 / 2}\right) .
\end{gathered}
$$

Lemma 5.5. The remaining terms have the following asymptotic behavior

$$
\mathrm{I}_{r}=o\left(\varepsilon^{2}\right), \quad \mathrm{IV}_{r}=o\left(\varepsilon^{2}\right)
$$

and

$$
\mathrm{I}_{s}=o(1), \quad \mathrm{IV}_{s}=o(1)
$$

Lemma 5.6. Finally the linear term $-\partial_{X}\left(\sqrt{g h_{\varepsilon}} r\right)$ in the equation (5.16) has the asymptotic behavior

$$
-\partial_{X}\left(\sqrt{g h_{\varepsilon}} r\right)=-\sqrt{g h} \partial_{X}\left[\left(1-\frac{\varepsilon}{2 h} \beta\left(\frac{X}{\varepsilon}\right)-\frac{\varepsilon^{2}}{2 h}\left(a_{\beta}+\frac{1}{4 h} \mathrm{E}\left(\beta^{2}\right)\right)\right) r\right]+o\left(\varepsilon^{2}\right) .
$$

The proofs of these lemmas are the content of Section 5.4. Using these asymptotic results in system (5.14)(5.15), and retaining only the leading terms, it reduces to (5.16) (5.17), with possibly different parameter values. When the parameters are chosen appropriately, the asymptotic behavior of the equations matches that of the solutions and the consistency procedure is closed.

Theorem 5.7. The result of the consistency analysis is that the free parameters in equations (5.16) (5.17) are

$$
\begin{aligned}
& a_{K d V}=\frac{1}{2 h} a_{\beta}+\frac{1}{4 h^{2}} \mathrm{E}\left(\beta^{2}\right)+\frac{3 c_{1}}{8 h^{2} \sqrt{g h}} \mathrm{E}\left(\left(\partial_{x} \beta\right)^{2}\right), \\
& b=-\frac{7 c_{1}}{64 h^{3}} \mathrm{E}\left(\left(\partial_{x} \beta\right)^{3}\right) .
\end{aligned}
$$


The parameter $a_{K d V}$ represents an adjustment at $\mathcal{O}\left(\varepsilon^{2}\right)$ to the overall wavespeed, while the sign of $b$ governs the stability of solutions. In many cases, $b$ vanishes.

Proposition 5.8. If the statistics of the ensemble $(\Omega, \mathcal{M}, \mathrm{P})$ are reversible in $x$, then $b=0$.

By reversible, we mean that the inversion $x \rightarrow-x$ preserves the probability measure $\mathbf{P}$, implying that $\mathrm{E}\left(\left(\partial_{x} \beta\right)^{3}\right)=0$.

5.4. Proofs of the above lemmas. In the analysis of the numerous integrals that go in to this consistency result, it is convenient to use the bracket notation as shorthand for integrations;

$$
\langle f, g\rangle:=\iint_{\mathbb{R}^{2}} f(X, t) g(X, t) d X d t .
$$

Proof of Lemma 5.2: We first rewrite $\mathrm{II}_{r}$ as

$$
\mathrm{II}_{r}=\frac{\sqrt{g}}{2} \varepsilon^{3 / 2} \partial_{X}\left(\sqrt{h_{\varepsilon}}\right) s_{1}=\frac{\sqrt{g}}{2} \varepsilon^{3 / 2} \partial_{X}\left(\sqrt{h_{\varepsilon}}-\mathrm{E}\left(\sqrt{h_{\varepsilon}}\right)\right) s_{1} .
$$

For any test function $\varphi(X, t)$, we compute $\left\langle\varphi, \mathrm{II}_{r}\right\rangle$ by substituting the expression (5.25) for $s_{1}$. This gives two terms, the first being

$$
\varepsilon^{3 / 2} \frac{\sqrt{g}}{2}\left\langle\varphi, \partial_{X}\left(\sqrt{h_{\varepsilon}}-\mathrm{E}\left(\sqrt{h_{\varepsilon}}\right)\right) s_{1}^{0}\right\rangle=-\frac{\sqrt{g}}{2} \varepsilon^{3 / 2}\left\langle\left(\sqrt{h_{\varepsilon}}-\mathrm{E}\left(\sqrt{h_{\varepsilon}}\right)\right), \partial_{X}\left(s_{1}^{0} \varphi\right)\right\rangle .
$$

Since

$$
\mathrm{E}\left(\sqrt{h_{\varepsilon}}\right)=\sqrt{h}+\mathcal{O}\left(\varepsilon^{2}\right)
$$

and because

$$
\sqrt{h_{\varepsilon}}-\mathrm{E}\left(\sqrt{h_{\varepsilon}}\right)=\sqrt{h}\left(1-\frac{\varepsilon}{2 h} \beta\left(\frac{X}{\varepsilon}\right)\right)-\sqrt{h}+\mathcal{O}\left(\varepsilon^{2}\right)=-\frac{\varepsilon}{2 \sqrt{h}} \beta\left(\frac{X}{\varepsilon}\right)+\mathcal{O}\left(\varepsilon^{2}\right),
$$

the first term in $\left\langle\varphi, \mathrm{II}_{r}\right\rangle$ is of order $o\left(\varepsilon^{2}\right)$. The second term in the expression of $\left\langle\varphi, \mathrm{II}_{r}\right\rangle$ is

$$
A:=\frac{\sqrt{g}}{8 h}\left\langle\varphi, \partial_{X}\left(\sqrt{h_{\varepsilon}}-\mathrm{E}\left(\sqrt{h_{\varepsilon}}\right)\right) \int_{X}^{X+\sqrt{g h} t} \partial_{x} \beta\left(\frac{\theta}{\varepsilon}\right) r\left(\theta, t+\frac{X-\theta}{\sqrt{g h}}\right) d \theta\right\rangle .
$$

By integration by parts,

$$
\begin{aligned}
A= & -\frac{\sqrt{g}}{8 h}\left\langle\partial_{X} \varphi,\left(\sqrt{h_{\varepsilon}}-\mathrm{E}\left(\sqrt{h_{\varepsilon}}\right)\right) \int_{X}^{X+\sqrt{g h} t} \partial_{x} \beta\left(\frac{\theta}{\varepsilon}\right) r\left(\theta, t+\frac{X-\theta}{\sqrt{g h}}\right) d \theta\right\rangle \\
& -\frac{\sqrt{g}}{8 h}\left\langle\varphi,\left(\sqrt{h_{\varepsilon}}-\mathrm{E}\left(\sqrt{h_{\varepsilon}}\right)\right) \frac{1}{\sqrt{g h}} \int_{X}^{X+\sqrt{g h} t} \partial_{x} \beta\left(\frac{\theta}{\varepsilon}\right) \partial_{t} r\left(\theta, t+\frac{X-\theta}{\sqrt{g h}}\right) d \theta\right\rangle \\
& -\frac{\sqrt{g}}{8 h}\left\langle\varphi,\left(\sqrt{h_{\varepsilon}}-\mathrm{E}\left(\sqrt{h_{\varepsilon}}\right)\right)\left[\partial_{x} \beta\left(\frac{X+\sqrt{g h} t}{\varepsilon}\right) r^{0}(X+\sqrt{g h} t)-\partial_{x} \beta\left(\frac{X}{\varepsilon}\right) r(X, t)\right]\right\rangle \\
= & -\frac{\sqrt{g}}{8 h}\left\langle\left(\partial_{X}-\frac{1}{\sqrt{g h}} \partial_{t}\right) \varphi,\left(\sqrt{h_{\varepsilon}}-\mathrm{E}\left(\sqrt{h_{\varepsilon}}\right)\right) \int_{X}^{X+\sqrt{g h} t} \partial_{x} \beta\left(\frac{\theta}{\varepsilon}\right) r\left(\theta, t+\frac{X-\theta}{\sqrt{g h}}\right) d \theta\right\rangle \\
& +\frac{\sqrt{g}}{8 h}\left\langle\varphi,\left(\sqrt{h_{\varepsilon}}-\mathrm{E}\left(\sqrt{h_{\varepsilon}}\right)\right) \partial_{x} \beta\left(\frac{X}{\varepsilon}\right) r(X, t)\right\rangle \\
\equiv & A_{1}+A_{2} .
\end{aligned}
$$

Analyze the second term first,

$$
A_{2}=-\frac{1}{32 h} \sqrt{\frac{g}{h}} \varepsilon\left\langle\varphi, \partial_{x}\left(\beta^{2}\right) r\right\rangle
$$


Replacing $r$ by its expression (5.26),

$$
\begin{gathered}
A_{2}=-\frac{1}{64 h^{2}} \sqrt{\frac{g}{h}} \varepsilon\left\langle\varphi, \partial_{x}\left(\beta^{2}\right)\left[\varepsilon^{2} \partial_{X} q \int_{\frac{X-\sqrt{g h t}}{\varepsilon}}^{\frac{X}{\varepsilon}} \beta\left(t^{\prime}\right) d t^{\prime}\right.\right. \\
\left.\left.+\varepsilon q\left(\beta\left(\frac{X}{\varepsilon}\right)-\beta\left(\frac{X-\sqrt{g h t} t}{\varepsilon}\right)\right)\right]\right\rangle+\mathcal{O}\left(\varepsilon^{\frac{5}{2}}\right) .
\end{gathered}
$$

By integration by parts, the first term of $A_{2}$ is $o\left(\varepsilon^{2}\right)$. The second to the last term of $A_{2}$ can be rewritten as

$$
-\frac{1}{64 h^{2}} \sqrt{\frac{g}{h}} \varepsilon^{2}\left\langle\varphi, \partial_{x}\left(\frac{2}{3} \beta^{3}\right) q\right\rangle
$$

which again by integration by parts contributes to $o\left(\varepsilon^{2}\right)$. The last term of $A_{2}$ contributes only $o\left(\varepsilon^{2}\right)$ due to Lemma 3.7. Now turn to $A_{1}$.

$$
\begin{aligned}
A_{1}= & -\frac{1}{16 h} \sqrt{\frac{g}{h}} \varepsilon^{2}\left\langle\left(\partial_{X}-\frac{1}{\sqrt{g h}} \partial_{t}\right) \varphi, \beta\left(\frac{X}{\varepsilon}\right) \int_{X}^{X+\sqrt{g h} t} \beta\left(\frac{\theta}{\varepsilon}\right) \frac{d}{d \theta} r\left(\theta, t+\frac{X-\theta}{\sqrt{g h}}\right) d \theta\right\rangle \\
& +\frac{1}{16 h} \sqrt{\frac{g}{h}} \varepsilon^{2}\left\langle\left(\partial_{X}-\frac{1}{\sqrt{g h}} \partial_{t}\right) \varphi, \beta\left(\frac{X}{\varepsilon}\right)\left[\beta\left(\frac{X+\sqrt{g h} t}{\varepsilon}\right) r^{0}(X+\sqrt{g h} t)-\beta\left(\frac{X}{\varepsilon}\right) r(X, t)\right]\right\rangle \\
& +o\left(\varepsilon^{2}\right) .
\end{aligned}
$$

The first term in the second line of $A_{1}$ is $o\left(\varepsilon^{2}\right)$ due to Lemma 3.7. The last term of $A_{1}$ is

$$
\begin{gathered}
-\frac{\varepsilon^{2}}{16 h} \sqrt{\frac{g}{h}} \mathrm{E}\left(\beta^{2}\right)\left\langle\left(\partial_{X}-\frac{1}{\sqrt{g h}} \partial_{t}\right) \varphi, q\right\rangle+o\left(\varepsilon^{2}\right) \\
=\frac{\varepsilon^{2}}{8 h} \sqrt{\frac{g}{h}} \mathrm{E}\left(\beta^{2}\right)\left\langle\varphi, \partial_{X} q\right\rangle+o\left(\varepsilon^{2}\right),
\end{gathered}
$$

leading to a contribution to $\mathrm{II}_{r}$ of

$$
\frac{\varepsilon^{2}}{8 h} \sqrt{\frac{g}{h}} \mathrm{E}\left(\beta^{2}\right) \partial_{X} r(X, t)+o\left(\varepsilon^{2}\right) .
$$

We now turn to the first term of $A_{1}$ which we denote $A_{3}$, and write it as

$$
A_{3}=-\frac{\varepsilon^{2}}{16 h} \sqrt{\frac{g}{h}}\left\langle\left(\partial_{X}-\frac{1}{\sqrt{g h}} \partial_{t}\right) \varphi, \beta\left(\frac{X}{\varepsilon}\right) \int_{X}^{X+\sqrt{g h} t} \beta\left(\frac{\theta}{\varepsilon}\right)\left(\partial_{X}-\frac{1}{\sqrt{g h}} \partial_{t}\right) r\left(\theta, t+\frac{X-\theta}{\sqrt{g h}}\right) d \theta\right\rangle .
$$

We express $\left(\partial_{X}-\frac{1}{\sqrt{g h}} \partial_{t}\right) r$ in terms of $q$ as

$$
\left(\partial_{X}-\frac{1}{\sqrt{g h}} \partial_{t}\right) r(X, t)=2 \partial_{X} q+\frac{1}{2 h}\left(\partial_{x} \beta\left(\frac{X}{\varepsilon}\right)-2 \partial_{x} \beta\left(\frac{X-\sqrt{g h} t}{\varepsilon}\right)\right) q+\mathcal{O}(\varepsilon) .
$$

Substitution of the above in $A_{3}$ gives rise to three terms, $(i),(i i)$, and (iii) which have the form (after we have dropped the constants):

$$
\begin{aligned}
& (i)=\varepsilon^{2}\left\langle\left(\partial_{X}-\frac{1}{\sqrt{g h}} \partial_{t}\right) \varphi, \beta\left(\frac{X}{\varepsilon}\right) \int_{X}^{X}+\sqrt{g h} t \beta\left(\frac{\theta}{\varepsilon}\right) \partial_{X} q\left(2 \theta-X-\sqrt{g h} t, \varepsilon^{2}\left(t+\frac{X-\theta}{\sqrt{g h}}\right)\right) d \theta\right\rangle \\
& (i i)=\varepsilon^{2}\left\langle\left(\partial_{X}-\frac{1}{\sqrt{g h}} \partial_{t}\right) \varphi, \beta\left(\frac{X}{\varepsilon}\right) \int_{X}^{X}+\sqrt{g h} t \frac{1}{2} \partial_{x}\left(\beta^{2}\left(\frac{\theta}{\varepsilon}\right)\right) q\left(2 \theta-X-\sqrt{g h} t, \varepsilon^{2}\left(t+\frac{X-\theta}{\sqrt{g h}}\right)\right) d \theta\right\rangle \\
& (i i i)=\varepsilon^{2}\left\langle\left(\partial_{X}-\frac{1}{\sqrt{g h}} \partial_{t}\right) \varphi,\right. \\
& \left.\quad \beta\left(\frac{X}{\varepsilon}\right) \int_{X}^{X+\sqrt{g h} t} \beta\left(\frac{\theta}{\varepsilon}\right) \partial_{x} \beta\left(\frac{2 \theta-X-\sqrt{g h t}}{\varepsilon}\right) q\left(2 \theta-X-\sqrt{g h} t, \varepsilon^{2}\left(t+\frac{X-\theta}{\sqrt{g h}}\right)\right) d \theta\right\rangle .
\end{aligned}
$$

The term $(i)$ is of the form

$$
\left\langle\int_{X}^{X+\sqrt{g h t}} \beta\left(\frac{X}{\varepsilon}\right) \beta\left(\frac{\theta}{\varepsilon}\right) \psi(\theta, X, t) d \theta\right\rangle .
$$


Applying Lemma 3.8, we show that this term is $\mathcal{O}\left(\varepsilon^{3}\right)$, and thus does not contribute to the limit of $\mathrm{II}_{r}$. By integration by parts, the term $(i i)$ is $\mathcal{O}\left(\varepsilon^{3}\right)$. Finally, for term (iii), we write $\partial_{x} \beta\left(\frac{2 \theta-X-\sqrt{g h} t}{\varepsilon}\right)=-\frac{\varepsilon}{\sqrt{g h}} \frac{d}{d t} \beta\left(\frac{2 \theta-X-\sqrt{g h t}}{\varepsilon}\right)$, leading to (iii) being again of order $\mathcal{O}\left(\varepsilon^{3}\right)$.

Proof of Lemma 5.3; Using that $h_{\varepsilon}=h-\varepsilon \beta\left(\frac{X}{\varepsilon}\right)+\mathcal{O}\left(\varepsilon^{2}\right)$

$$
\mathrm{II}_{s}=\frac{1}{4} \partial_{x} \beta\left(\frac{X}{\varepsilon}\right) \sqrt{\frac{g}{h}} \varepsilon^{-3 / 2}\left(1+\frac{\varepsilon}{2 h} \beta\left(\frac{X}{\varepsilon}\right)\right) r .
$$

Since $r(X, t)=q\left(X-\sqrt{g h} t, \varepsilon^{2} t\right)+\mathcal{O}(\varepsilon)$, the second term of (5.57) is

$$
\frac{\varepsilon^{-1 / 2}}{16 h} \sqrt{\frac{g}{h}} \partial_{x} \beta^{2}\left(\frac{X}{\varepsilon}\right)\left(q\left(X-\sqrt{g h} t, \varepsilon^{2} t\right)+\mathcal{O}(\varepsilon)\right)=\mathcal{O}\left(\varepsilon^{1 / 2}\right)
$$

due to Lemma 3.5. Compute the limit as $\varepsilon \rightarrow 0$ of $\mathrm{II}_{s}$. Substituting the expression (5.26) for $r$, we get, for any test function $\varphi(x, t)$

$$
\begin{aligned}
\left\langle\varphi, \frac{\varepsilon^{-\frac{3}{2}}}{4} \sqrt{\frac{g}{h}} \partial_{x} \beta\left(\frac{X}{\varepsilon}\right) r\right\rangle= & \frac{\varepsilon^{-\frac{3}{2}}}{4} \sqrt{\frac{g}{h}}\left\langle\varphi, \partial_{x} \beta\left(\frac{X}{\varepsilon}\right) q\right\rangle \\
& +\frac{\varepsilon^{-\frac{1}{2}}}{4} \sqrt{\frac{g}{h}}\left\langle\varphi, \partial_{x} \beta\left(\frac{X}{\varepsilon}\right) \partial_{X} q \frac{\varepsilon}{2 h} \int_{\frac{X}{\varepsilon} \sqrt{g h t}}^{\frac{X}{\varepsilon}} \beta\left(t^{\prime}\right) d t^{\prime}\right\rangle \\
& +\frac{\varepsilon^{-\frac{3}{2}}}{4} \sqrt{\frac{g}{h}}\left\langle\varphi, \partial_{x} \beta\left(\frac{X}{\varepsilon}\right) \frac{\varepsilon}{2 h} q\left(\beta\left(\frac{X}{\varepsilon}\right)-\beta\left(\frac{X-\sqrt{g h} t}{\varepsilon}\right)\right)\right\rangle .
\end{aligned}
$$

The first term of the RHS of (5.59) tends to the first term of (5.35) by application of Lemma 3.5. The second term of (5.59) is rewritten, by integration by parts, as

$$
\begin{aligned}
-\frac{\varepsilon^{\frac{1}{2}}}{8 h} & \sqrt{\frac{g}{h}}\left\langle\partial_{X}\left(\varphi \partial_{X} q\right), \beta\left(\frac{X}{\varepsilon}\right) \int_{(X-\sqrt{g h} t) / \varepsilon}^{\frac{X}{\varepsilon}} \beta\left(t^{\prime}\right) d t^{\prime}\right\rangle-\frac{\varepsilon^{\frac{1}{2}}}{8 h} \sqrt{\frac{g}{h}}\left\langle\varphi, \beta^{2}\left(\frac{X}{\varepsilon}\right) \partial_{X} q\right\rangle \\
& +\frac{\varepsilon^{\frac{1}{2}}}{8 h} \sqrt{\frac{g}{h}}\left\langle\varphi, \beta\left(\frac{X}{\varepsilon}\right) \beta\left(\frac{X-\sqrt{g h t}}{\varepsilon}\right) \partial_{X} q\right\rangle .
\end{aligned}
$$

Clearly all terms are $o(1)$. The third term of (5.59) is rewritten

$$
\frac{\varepsilon^{-\frac{1}{2}}}{16 h} \sqrt{\frac{g}{h}}\left\langle\varphi, \partial_{x} \beta^{2}\left(\frac{X}{\varepsilon}\right) q\right\rangle-\frac{\varepsilon^{-\frac{1}{2}}}{8 h} \sqrt{\frac{g}{h}}\left\langle\varphi, \beta\left(\frac{X}{\varepsilon}\right) \partial_{x} \beta\left(\frac{X-\sqrt{g h} t}{\varepsilon}\right) q\right\rangle,
$$

which is $o(1)$ by application of Lemmas 3.5 and 3.7 .

Proof of Lemma 5.4: Decompose $\mathrm{III}_{r}$ as the sum of the two terms

$$
\begin{aligned}
& C=\frac{c_{1}}{4} \varepsilon^{2} \frac{\partial_{x} \beta\left(\frac{X}{\varepsilon}\right)}{h_{\varepsilon}} \partial_{X}^{2} r, \\
& D=-\frac{c_{1}}{4} \varepsilon^{2+3 / 2} \frac{\partial_{x} \beta\left(\frac{X}{\varepsilon}\right)}{h_{\varepsilon}} \partial_{X}^{2} s_{1} .
\end{aligned}
$$

We compute $\partial_{X}^{2} r$ from (5.26) and do not write terms that will clearly give a contribution of $o\left(\varepsilon^{2}\right)$. We get

$$
\langle\varphi, C\rangle=\frac{c_{1}}{8 h} \varepsilon^{2}\left\langle\varphi, \frac{\partial_{x} \beta}{h_{\varepsilon}}\left(3 \partial_{X} q \partial_{x} \beta+\varepsilon^{-1} q \partial_{x}^{2} \beta\right)\right\rangle+o\left(\varepsilon^{2}\right) .
$$

The first term in (5.63), denoted $C_{1}$ is

$$
\left\langle\varphi, C_{1}\right\rangle=\frac{3 c_{1}}{8 h^{2}} \varepsilon^{2} \mathrm{E}\left(\left(\partial_{x} \beta\right)^{2}\right)\left\langle\varphi, \partial_{X} q\left(X-\sqrt{g h} t, \varepsilon^{2} t\right)\right\rangle .
$$


We substitute $\partial_{X} q$ in terms of $r$ in $C_{1}$ using (5.26) and we write

$$
\partial_{X} q=\partial_{X} r-\frac{q}{2 h}\left(\partial_{x} \beta\left(\frac{X}{\varepsilon}\right)-\partial_{x} \beta\left(\frac{X-\sqrt{g h} t}{\varepsilon}\right)\right)+\mathcal{O}(\varepsilon) .
$$

We then conclude that $C_{1}$ can be written as a functional of $r$ as

$$
\left\langle\varphi, C_{1}\right\rangle=\frac{3 c_{1}}{8 h^{2}} \varepsilon^{2} \mathrm{E}\left(\left(\partial_{x} \beta\right)^{2}\right)\left\langle\varphi, \partial_{X} r\right\rangle .
$$

The second term in $C$, denoted by $C_{2}$ is

$$
\begin{aligned}
\left\langle\varphi, C_{2}\right\rangle & =\frac{c_{1}}{16 h} \varepsilon\left\langle\varphi, \frac{1}{h_{\varepsilon}} \partial_{x}\left(\partial_{x} \beta\right)^{2} q\right\rangle \\
& =\frac{c_{1}}{16 h^{2}} \varepsilon\left\langle\varphi, \partial_{x}\left(\partial_{x} \beta\right)^{2}\left(1+\frac{\varepsilon}{h} \beta\right) q\right\rangle+\mathcal{O}\left(\varepsilon^{3}\right) \\
& =\frac{c_{1}}{16 h^{3}}\left\langle\varphi, \varepsilon^{2} \partial_{x}\left(\partial_{x} \beta\right)^{2} \beta q\right\rangle+o\left(\varepsilon^{2}\right) \\
& =-\frac{c_{1}}{16 h^{3}} \varepsilon^{2} \mathrm{E}\left(\left(\partial_{x} \beta\right)^{3}\right)\langle\varphi, q\rangle+o\left(\varepsilon^{2}\right) .
\end{aligned}
$$

We conclude that the term $C$ of $\operatorname{III}_{r}$ is

$$
C=\frac{3 c_{1}}{8 h^{2}} \varepsilon^{2} \mathrm{E}\left(\left(\partial_{x} \beta\right)^{2}\right) \partial_{X} r-\frac{c_{1}}{16 h^{3}} \varepsilon^{2} \mathrm{E}\left(\left(\partial_{x} \beta\right)^{3}\right) r+o\left(\varepsilon^{2}\right) .
$$

We compute the term $D$ of $\operatorname{III}_{r}$ given in (5.62). For this, we compute $\partial_{X}^{2} s_{1}$ in terms of $r$ and get:

$$
\begin{aligned}
\partial_{X}^{2} s_{1} & (X, t)=\partial_{X}^{2} s_{1}^{0}(X+\sqrt{g h} t)+\frac{1}{4 h} \varepsilon^{-\frac{3}{2}}\left[\frac{1}{\varepsilon} \partial_{x}^{2} \beta\left(\frac{X+\sqrt{g h} t}{\varepsilon}\right) r^{0}(X+\sqrt{g h} t)\right. \\
& +\partial_{x} \beta\left(\frac{X+\sqrt{g h} t}{\varepsilon}\right) \partial_{X} r^{0}(X+\sqrt{g h} t)-\frac{1}{\varepsilon} \partial_{x}^{2} \beta\left(\frac{X}{\varepsilon}\right) r(X, t)-\partial_{x} \beta\left(\frac{X}{\varepsilon}\right) \partial_{X} r(X, t) \\
& +\frac{1}{\sqrt{g h}} \partial_{x} \beta\left(\frac{X+\sqrt{g h t} t}{\varepsilon}\right) \partial_{t} r(X+\sqrt{g h} t, 0)-\frac{1}{\sqrt{g h}} \partial_{x} \beta\left(\frac{X}{\varepsilon}\right) \partial_{t} r(X, t) \\
& \left.+\frac{1}{g h} \int_{X}^{X+\sqrt{g h t} t} \partial_{x} \beta\left(\frac{\theta}{\varepsilon}\right) \partial_{t t} r\left(\theta, t+\frac{X-\theta}{\sqrt{g h}}\right) d \theta\right] .
\end{aligned}
$$

All terms containing the process $\beta$ or its derivatives at two different points $X / \varepsilon$ and $(X+\sqrt{g h t}) / \varepsilon$ will not contribute because of Lemma 3.7. The term containing $s_{1}^{0}$ will be $o\left(\varepsilon^{2}\right)$. The remaining terms that need attention are

$$
\begin{gathered}
\frac{c_{1} \varepsilon^{2}}{16 h}\left\langle\varphi, \frac{\partial_{x} \beta}{h_{\varepsilon}}\left[\varepsilon^{-1} \partial_{x}^{2} \beta r(X, t)+\partial_{x} \beta\left(\partial_{X} r(X, t)+\frac{1}{\sqrt{g h}} \partial_{t} r(X, t)\right)\right]\right\rangle \\
-\frac{c_{1} \varepsilon^{2}}{16} \frac{1}{g h^{2}}\left\langle\varphi, \frac{\partial_{x} \beta}{h_{\varepsilon}} \int_{X}^{X+\sqrt{g h t}} \partial_{x} \beta\left(\frac{\theta}{\varepsilon}\right) \partial_{t t} r\left(\theta, t+\frac{X-\theta}{\sqrt{g h}}\right) d \theta\right\rangle .
\end{gathered}
$$

Noting that $\partial_{X} r+\frac{1}{\sqrt{g h}} \partial_{t} r=\mathcal{O}(\varepsilon)$, we have that

$$
\frac{c_{1} \varepsilon^{2}}{16 h}\left\langle\varphi, \frac{\partial_{x} \beta}{h_{\varepsilon}}\left(\partial_{x} \beta\left(\partial_{X} r(X, t)+\frac{1}{\sqrt{g h}} \partial_{t} r(X, t)\right)\right)\right\rangle=\mathcal{O}\left(\varepsilon^{3}\right) .
$$

The first term in (5.70) has the form

$$
\begin{aligned}
& \frac{c_{1} \varepsilon}{32 h^{2}}\left\langle\varphi,\left(1+\frac{\varepsilon}{h} \beta\left(\frac{X}{\varepsilon}\right)\right) \partial_{x}\left(\left(\partial_{x} \beta\right)^{2}\right) r\right\rangle+o\left(\varepsilon^{2}\right) \\
& \quad=\frac{c_{1} \varepsilon}{32 h^{2}}\left\langle\varphi, \partial_{x}\left(\left(\partial_{x} \beta\right)^{2}-\mathrm{E}\left(\left(\partial_{x} \beta\right)^{2}\right)\right) r\right\rangle-\frac{c_{1} \varepsilon^{2}}{32 h^{3}}\left\langle\varphi, \mathrm{E}\left(\left(\partial_{x} \beta\right)^{3}\right) r\right\rangle+o\left(\varepsilon^{2}\right) .
\end{aligned}
$$


Integrating by parts the first term of (5.72), we get two contributions; when the derivative acts on $\varphi$, it is $o\left(\varepsilon^{2}\right)$ using Lemma 3.5 and the fact that $r=q+\mathcal{O}(\varepsilon)$. When the derivative acts on $r$, we get:

$$
-\frac{c_{1} \varepsilon^{2}}{32 h^{2}}\left\langle\varphi,\left(\left(\partial_{x} \beta\right)^{2}-\mathrm{E}\left(\left(\partial_{x} \beta\right)^{2}\right)\right) \partial_{X} r\right\rangle
$$

Here we replace $\partial_{X} r$ by its expression in terms of $q$ :

$$
\partial_{X} r=\partial_{X} q+\frac{1}{2 h} q\left(\partial_{x} \beta\left(\frac{X}{\varepsilon}\right)-\partial_{x} \beta\left(\frac{X-\sqrt{g h} t}{\varepsilon}\right)\right)+\mathcal{O}(\varepsilon) .
$$

The resulting contribution for (5.73) is

$$
-\frac{c_{1} \varepsilon^{2}}{32 h^{2}}\left\langle\varphi,\left(\left(\partial_{x} \beta\right)^{2}-\mathrm{E}\left(\left(\partial_{x} \beta\right)^{2}\right)\right) \partial_{X} r\right\rangle=-\frac{c_{1} \varepsilon^{2}}{64 h^{3}}\left\langle\varphi, \mathrm{E}\left(\left(\partial_{x} \beta\right)^{3}\right) r\right\rangle+o\left(\varepsilon^{2}\right) .
$$

The last term to consider is the fourth term of (5.70) where the derivatives with respect to $t$ can be moved outside the integral using the fact that

$$
\begin{aligned}
\int_{X}^{X} & +\sqrt{g h t} \partial_{x} \beta\left(\frac{\theta}{\varepsilon}\right) \partial_{t t} r\left(\theta, t+\frac{X-\theta}{\sqrt{g h}}\right) d \theta=\partial_{t t} \int_{X}^{X+\sqrt{g h} t} \partial_{x} \beta\left(\frac{\theta}{\varepsilon}\right) r\left(\theta, t+\frac{X-\theta}{\sqrt{g h}}\right) d \theta \\
& -\sqrt{g h} \partial_{x} \beta\left(\frac{X+\sqrt{g h t} t}{\varepsilon}\right) \partial_{t} r(X+\sqrt{g h} t, 0)-g h \partial_{x} \beta\left(\frac{X+\sqrt{g h} t}{\varepsilon}\right) \partial_{X} r(X+\sqrt{g h} t, 0) \\
& -\frac{g h}{\varepsilon} \partial_{x x} \beta\left(\frac{X+\sqrt{g h} t}{\varepsilon}\right) r(X+\sqrt{g h} t, 0) .
\end{aligned}
$$

Using Lemma 3.7 again,

$$
\begin{aligned}
& -\frac{c_{1} \varepsilon^{2}}{16} \frac{1}{g h^{2}}\left\langle\varphi, \frac{\partial_{x} \beta}{h_{\varepsilon}} \int_{X}^{X+\sqrt{g h} t} \partial_{x} \beta\left(\frac{\theta}{\varepsilon}\right) \partial_{t t} r\left(\theta, t+\frac{X-\theta}{\sqrt{g h}}\right) d \theta\right\rangle \\
& \quad=-\frac{c_{1} \varepsilon^{2}}{16} \frac{1}{g h^{2}}\left\langle\partial_{t t} \varphi, \frac{\partial_{x} \beta}{h_{\varepsilon}} \int_{X}^{X+\sqrt{g h t}} \partial_{x} \beta\left(\frac{\theta}{\varepsilon}\right) r\left(\theta, t+\frac{X-\theta}{\sqrt{g h}}\right) d \theta\right\rangle+o\left(\varepsilon^{2}\right) .
\end{aligned}
$$

Using the derivative in the first factor of $\partial_{x} \beta$ appearing in the above expression and integrating by parts leads to the appearance of an additional $\varepsilon$, making the expression $\mathcal{O}\left(\varepsilon^{3}\right)$. We have obtained that

$$
D=-\frac{3 c_{1} \varepsilon^{2}}{64 h^{3}} \mathrm{E}\left(\left(\partial_{x} \beta\right)^{3}\right) r+o\left(\varepsilon^{2}\right) .
$$

Adding the expression for $C$ and $D$, we have shown that (5.36) describes the asymptotic behavior of $\mathrm{III}_{\mathrm{r}}$.

Proof of Lemma 5.5: Following the criterion of Section 3, these terms are integrated against test functions $\varphi$, and derivatives can be moved to $\varphi$ by integration by parts.

Proof of Lemma 5.6. The regularized depth $h_{\varepsilon}$ is defined as $h_{\varepsilon}(X)=h-\varepsilon \beta\left(\frac{X}{\varepsilon}\right)-$ $\varepsilon^{2} a_{\beta}$. Thus the regularized linear wave speed is

$$
\sqrt{g h_{\varepsilon}}=\sqrt{g h}\left(1-\frac{\varepsilon}{2 h} \beta-\varepsilon^{2} \frac{a_{\beta}}{2 h}-\varepsilon^{2} \frac{\beta^{2}}{8 h^{2}}\right)+o\left(\varepsilon^{2}\right) .
$$

The term $\left\langle\varphi, \partial_{X}\left(\beta^{2} r\right)\right\rangle$ is calculated as

$$
\left\langle\varphi, \partial_{X}\left(\beta^{2} r\right)\right\rangle=\left\langle\varphi, \mathrm{E}\left(\beta^{2}\right) \partial_{X} r\right\rangle-\left\langle\partial_{X} \varphi,\left(\beta^{2}-\mathrm{E}\left(\beta^{2}\right)\right) r\right\rangle .
$$

Using that $r=q+\mathcal{O}(\varepsilon)$, we get that the second term in (5.80) is $\mathcal{O}(\sqrt{\varepsilon})$. 


\section{REMARKS ON THE EXPECTATION OF SOLUTIONS}

It is normal to calculate $\mathrm{E}(r(X, t, \omega))=p(X, t)$ as a basic prediction of the solution $r(X, t, \omega)$ itself. We remark that $r(X, t, \omega)$ is a realization dependent function where the randomness manifests itself on the same level as dispersive and nonlinear effects. In the paper [21] on apparent diffusion, the authors present an analysis of the function $p(X, t)$ in the case of the linear water wave problem with bottom given by $\{y=-h+\sqrt{\varepsilon} \beta(X / \varepsilon)\}$. In the fully nonlinear regime of the present paper, diffusion is weaker, and occurs only on time scales larger than those of $\mathcal{O}(1)$ in $\mathrm{KdV}$ time $\tau$, as the following calculation shows.

In the sense of weak limits of probability measures, as $\varepsilon \rightarrow 0$,

$$
r(X, t)=q(Y, \tau),
$$

where

$$
Y=X-\sqrt{g h} t+\frac{\varepsilon^{3 / 2}}{2 h}(g h)^{1 / 4} \sigma_{\beta} B(t)+\varepsilon^{2} a_{K d V} \sqrt{g h} t, \quad \text { and } \quad \tau=\varepsilon^{2} t .
$$

Compute the expectation of the main component of the solution $r$ :

$$
\begin{gathered}
\mathrm{E}(r(X, t))=\int_{-\infty}^{\infty} q\left(X-\sqrt{g h} t+\frac{\varepsilon^{3 / 2}}{2 h} \sigma_{\beta}(g h)^{1 / 4} u+\varepsilon^{2} a_{K d V} \sqrt{g h} t, \tau\right) d \mu_{B(t)}(u) \\
\quad=\frac{1}{\sqrt{2 \pi t}} \int_{-\infty}^{\infty} q\left(X-\sqrt{g h} t+\frac{\varepsilon^{3 / 2}}{2 h} \sigma_{\beta}(g h)^{\frac{1}{4}} u+\varepsilon^{2} a_{K d V} \sqrt{g h} t, \tau\right) \mathrm{e}^{-\frac{u^{2}}{2 t}} d u
\end{gathered}
$$

Assuming that $\max _{\tau}|q(., \tau)|_{L^{1}}<\infty$, we have for fixed t,

$$
\begin{aligned}
\max _{X} \mathrm{E}(r(X, t)) \leq \max _{X^{\prime}} \frac{1}{\sqrt{2 \pi t}} \int_{-\infty}^{\infty}\left|q\left(X^{\prime}+\frac{\varepsilon^{3 / 2}}{2 h} \sigma_{\beta}(g h)^{\frac{1}{4}} u, \tau\right)\right| d u \\
\leq \frac{2 h \varepsilon^{-\frac{3}{2}}}{\sqrt{2 \pi t}}(g h)^{-\frac{1}{4}} \int_{-\infty}^{\infty}|q(v, \tau)| d v .
\end{aligned}
$$

This time decay of order $\varepsilon^{-3 / 2} t^{-1 / 2}=(\varepsilon \tau)^{-1 / 2}$ shows that the diffusion coefficient is of order $\mathcal{O}(\varepsilon)$, meaning that diffusion effects occur at an order higher that the one considered for the derivation of the KdV equation. To observe diffusion created by random effect at the order of the relevant terms for the $\mathrm{KdV}$ would require a scaling for the bottom variations of the form $-h+\sqrt{\varepsilon} \beta(x, \omega)$, which is a 'rougher' bottom that the one considered in this paper. This is the natural scaling that was considered in the linear analysis of [21. However, such a hypothesis also affects the nonlinear and dispersive nature of solutions and indeed it will introduce additional terms in the nonlinear coupled system of equations for $(r, s)$ that would have to be taken into account. This is beyond the scope of the present paper and is planned as the focus of a subsequent study.

\section{ACKNOWLEDGMENTS}

WC would like to thank S. R. S. Varadhan for his suggestions at the beginning of this project. WC has been partially supported by the Canada Research Chairs Program and NSERC through grant number 238452-01, ODE by a CRC postdoctoral fellowship, PG by the University of Delaware Research Foundation and NSF through grant number DMS-0625931, and CS by NSERC through grant number 46179-05. 


\section{REFERENCES}

[1] Alvarez-Samaniego, B., Lannes, D. Large time existence for 3D water waves and asymptotics, Preprint, 2007.

[2] Artiles, W. and Nachbin, A., Asymptotic nonlinear wave modeling through the Dirichlet-toNeumann operator., Methods Appl. Anal. 11 (2004), no. 4, 475-492.

[3] Billingsley, P., Convergence of probability measures, John Wiley \& Sons, Inc., New YorkLondon-Sydney (1968).

[4] Bona, J., Chen, M. \& Saut, J.-C., Boussinesq equations and other systems for smallamplitude long waves in nonlinear dispersive media. I. Derivation and linear theory, J. Nonlinear Sci. 12 (2002), 283-318.

[5] Bona, J., Colin, T., \& Lannes, D., Long wave approximations for water waves, Arch. Ration. Mech. Anal. 17 (2005), 373-410.

[6] Chazel, F., Influence of bottom topography on long water waves, Preprint, 2007.

[7] Craig, W., An existence theory for water waves and the Boussinesq and Korteweg-de Vries scaling limits, Comm. P. Diff. Eq. 8(1985), pp. 787-1003.

[8] Craig, W., Guyenne, P. and Kalisch, H., Hamiltonian long-wave expansions for free surfaces and interfaces. Comm. Pure Appl. Math. 58 (2005), 1587-1641.

[9] Craig, W., Guyenne, P., Nicholls, D. and Sulem, C., Hamiltonian long-wave expansions for water waves over a rough bottom. Proc. R. Soc. Lond. Ser. A Math. Phys. Eng. Sci. 461 (2005), no. 2055, 839-873.

[10] Craig, W. and Sulem, C. , Numerical simulation of gravity waves. J. Comput. Phys. 108 (1993), no. 1, 73-83.

[11] Craig, W., Sulem, C. and Sulem, P.-L. Nonlinear modulation of gravity waves: a rigorous approach, Nonlinearity 5 (1992), no. 2, 497-522.

[12] Cramer H. and Leadbetter M.R., Stationary and Related Stochastic Processes, John Wiley and Sons, Inc. New York - London - Sydney, 1967.

[13] Doukhan, P. Mixing Properties and Examples, Lecture Notes in Statistics 85, SpringerVerlag, 1994.

[14] Grataloup, G. and Mei, C. C., Long waves in shallow water over a random seabed, Phys. Rev. E 68 (2003), 026314.

[15] Howe, M.S., On wave scattering by random inhomogeneities, with application to the theory of weak bores, J. Fluid. Mech. 45 (1971), 785-804.

[16] Kano, T. \& Nishida, T. A mathematical justification for Korteweg-de Vries equation and Boussinesq equation of water surface waves, Osaka J. Math. 23 (1986), 389-413.

[17] Mei, C. C. and Hancock, M. Weakly nonlinear surface waves over a random seabed, J. Fluid Mech. 475 (2003), 247-268.

[18] Mei, C. C. and Li, Y., Evolution of solitons over a randomly rough seabed, Phys. Rev. E (3) 70 (2004), no. 1, 016302.

[19] Nachbin, A. The localization length of randomly scattered water waves, J. Fluid Mech. 296 (1995), 353-372.

[20] Nachbin, A., A terrain-following Boussinesq system, SIAM J. Appl. Math. 63 (2003), no. 3, 905-922.

[21] Nachbin, A. and Sølna, K. Apparent diffusion due to topographic microstructure in shallow waters, Phys. Fluids 15 (2003), no. 1, 66-77.

[22] Oodaira, H. and Yoshihara, K. Functional central limit theorems for strictly stationary processes satisfying the strong mixing condition, Kōdai Math. Sem. Rep. 24 1972, 259-269.

[23] Pihl, J. H., Mei, C. C. and Hancock, M. Surface gravity waves over a two-dimensional random seabed. Phys. Rev. E 66 (2002), 016611.

[24] Rosales, R. and Papanicolaou, G., Gravity waves in a channel with a rough bottom. Stud. Appl. Math. 68 (1983), no. 2, 89-102.

[25] Schneider, G. \& Wayne, C.E., The long-wave limit for the water wave problem. I. The case of zero surface tension, Comm. Pure Appl. Math. 53 (2000), 1475-1535.

[26] Sølna, K. and Papanicolaou, G., Ray theory for a locally layered random medium, Waves Random Media 18 2000, 151-198.

[27] Strassen, V. and Dudley, R. M., The central limit theorem and $\varepsilon$-entropy, 1969 Probability and Information Theory, 224-231. Lectures Notes in Mathematics, Springer, Berlin. 
[28] Wright, J.D., Corrections to the KdV approximation for water waves, SIAM J. Math. Anal. 37 (2005), 1161-1206.

[29] Yosihara, H., Gravity waves on the free surface of an incompressible perfect fluid of finite depth, Publ. Res. Inst. Math. Sci. 18 (1982), no. 1, 49-96.

[30] Zakharov, V. E., Stability of periodic waves of finite amplitude on the surface of a deep fluid. J. Appl. Mech. Tech. Phys. 9 (1968), 1990-1994.

${ }^{1}$ Centre de Mathématiques Appliquées, Ecole Polytechnique, Route de Saclay, 91128 Palaiseau Cedex France

2 Department of Mathematics and Statistics, McMaster University, 1280 Main St. West, Hamilton, ON L8S4K1, Canada

3 Department of Mathematics, University of Delaware, 501 Ewing Hall Newark, DE 19716-2553, USA

4 Department of Mathematics, University of Toronto, 40 St George St, Toronto, ON M5S2E4, CANADA 